OPEN ACCESS

Edited by:

Farhad Maleki,

McGill University, Canada

Reviewed by:

Marc Gregory Yu,

Joslin Diabetes Center and Harvard

Medical School, United States

Yin Detao,

First Affiliated Hospital of Zhengzhou

University, China

${ }^{*}$ Correspondence:

Zhen Liu

liuzhen1973@aliyun.com

Specialty section:

This article was submitted to

Computational Genomics,

a section of the journal

Frontiers in Genetics

Received: 07 November 2021

Accepted: 14 January 2022

Published: 09 February 2022

Citation:

Tan H, Wang L and Liu Z (2022) Role of Suprabasin in the Dedifferentiation of Follicular Epithelial Cell-Derived Thyroid Cancer and Identification of Related Immune Markers.

Front. Genet. 13:810681. doi: 10.3389/fgene.2022.810681

\section{Role of Suprabasin in the} Dedifferentiation of Follicular Epithelial Cell-Derived Thyroid Cancer and Identification of Related Immune Markers

\author{
Hao Tan, Lidong Wang and Zhen Liu* \\ Department of General Surgery, Shengjing Hospital of China Medical University, Shenyang, China
}

Background: Aberrant regulation of suprabasin (SBSN) is associated with the development of cancer and immune disorders. SBSN influences tumor cell migration, proliferation, angiogenesis, and immune resistance. In this study, we investigated the potential correlation between SBSN expression and immune infiltration in thyroid cancer.

Methods: The expression of SBSN in 80 papillary thyroid carcinoma (PTC) specimens was determined using quantitative reverse-transcription polymerase chain reaction, western blotting, and immunohistochemical staining. The expression of SBSN in 9 cases of poorly differentiated thyroid carcinoma (PDTC) and 18 cases of anaplastic thyroid carcinoma (ATC) was evaluated by immunohistochemical staining. Comprehensive bioinformatics analysis of SBSN expression was performed using The Cancer Genome Atlas and Gene Expression Omnibus datasets, and the relationship of SBSN expression with M2 macrophages and T regulatory cells (Tregs) in ATC and PTC was verified by immunohistochemical staining.

Results: Compared with those in adjacent normal tissues, the expression levels of SBSN mRNA and protein were significantly higher in PTC tissues. SBSN expression level was correlated with that of cervical lymph node metastasis in PTC patients. Immunohistochemical staining results showed statistically significant differences among high-positive expression rates of SBSN in PTC, PDTC, and ATC. Functional enrichment analysis showed that SBSN expression was associated with pathways related to cancer, cell signaling, and immune response. Furthermore, analysis of the tumor microenvironment (using CIBERSORT-ABS and $x$ Cell algorithms) showed that SBSN expression affected immune cell infiltration and the cancer immunity cycle, and immunohistochemistry

\footnotetext{
Abbreviations: ACC, adenoid cystic carcinoma; ATC, anaplastic thyroid carcinoma; CAF, cancer-associated fibroblast; DCs, dendritic cells; ESCC, esophageal squamous cell carcinoma; GSEA, gene set enrichment analysis; HSCs, hematopoietic stem cells; iDCs, immature dendritic cells; MDSCs, marrow-derived suppressor cells; MEP, megakaryocyte-erythroid progenitor; NSCLC, non-small cell lung cancer; PDTC, poorly differentiated thyroid carcinoma; PTC, papillary thyroid carcinomas; qRTPCR, quantitative reverse-transcription polymerase chain reaction; SBSN, suprabasin; ssGSEA, single-sample gene set enrichment analysis; TAMs, tumor-associated macrophages; TCGA, the cancer genome atlas; Th2, T helper cell type 2; TIICs, tumor-infiltrating immune cells; TIP, tumor immunophenotype; TME, tumor microenvironment; Tregs, regulatory T cells.
} 
confirmed a significant increase in M2 macrophage and Treg infiltration in tumor tissues with high-positive SBSN expression.

Conclusion: These findings reveal that SBSN may be involved in thyroid carcinogenesis, tumor dedifferentiation progression, and immunosuppression as an important regulator of tumor immune cell infiltration.

Keywords: suprabasin, thyroid cancer, lymph node metastasis, immune infiltration, tumor immunosuppression, dedifferentiation

\section{INTRODUCTION}

Thyroid cancer is one of the most common endocrine tumors and its incidence has increased globally over the last 30 years ( $\mathrm{La}$ Vecchia et al., 2015). Most thyroid cancers originate from the follicular epithelial cells of the thyroid gland, which secrete iodine-containing thyroid hormones. Follicular epitheliumderived thyroid cancers can be classified as papillary thyroid carcinoma (PTC), follicular thyroid carcinoma, poorly differentiated thyroid carcinoma (PDTC), and anaplastic thyroid carcinoma (ATC) (Dralle et al., 2015). Of these, PTC is the most common pathological type, accounting for $80-90 \%$ of all thyroid cancers (Abdullah et al., 2019). Most thyroid cancers exhibit inert biological behavior and have a good prognosis, with a 20-year survival rate of 95\% (Gospodarowicz et al., 2001). However, recurrence, metastasis, and resistance to radioiodine therapy in PDTC, ATC, and some invasive PTCs remain the leading causes of death from thyroid cancer (Mazzaferri and Jhiang, 1994; Molinaro et al., 2017; Xu and Ghossein, 2020), with more than $25 \%$ of patients with PTC experiencing recurrence during a long-term follow-up (Abdullah et al., 2019). Furthermore, a high rate (up to $85 \%$ ) of cervical-lymph-node metastasis, which is considered a very high-risk factor for PTC recurrence, has been documented in patients with PTC (Zheng et al., 2019). Currently, some studies suggest that ATC is different from PTC in the early stages of tumor development and that the two tumor types evolve via distinct mechanisms (Capdevila et al., 2018). However, it is also believed that histologically highly differentiated thyroid cancer may dedifferentiate into PDTC or ATC via a multi-step process of genetic and epigenetic alterations (Papp and Asa, 2015) or that ATC can develop from PTC via accumulation of genomic mutations (Landa et al., 2016).

Immunotherapy has long been a focus area in oncology and is effective against non-small cell lung cancer (NSCLC) and kidney cancer (Motzer et al., 2015; Reck et al., 2016). Immune cell infiltration of the tumor microenvironment (TME) has also been associated with survival in many patients with solid tumors (Baxevanis et al., 2019). Infiltrating immune cells may be used as drug targets to improve patient survival (Lote et al., 2015). A previous study showed that the polarization of a higher number of tumor-associated macrophages (TAMs) in a tumorpromoting M2 phenotype implies a poorer survival of patients with ATC (Jung et al., 2015). Fang et al. (2014) found that TAMs purified from human PTC could promote invasiveness of thyroid cancer cell lines by secreting CXCL8. Melillo et al. (2010) found that the density of tumor-associated mast cells was higher in PTC than in normal tissue and correlated with extra-thyroidal tumor infiltration. In PTC, the number of $\mathrm{CD} 4^{+} \mathrm{T}$ cells correlates with the tumor size, whereas that of Tregs correlates with lymph node metastasis (French et al., 2010). Tregs are enriched in tumorinvolved lymph nodes, and their numbers correlate with PTC recurrence (French et al., 2012). Tumor-infiltrating lymphocytes, TAMs, and tumor-infiltrating neutrophils influence the prognosis and efficacy of chemotherapy and immunotherapy (Waniczek et al., 2017; Zhang et al., 2018). In addition, Chen and Mellman (2013) divided the cancer immunity cycle into seven steps, including the release of cancer cell antigens, cancer antigen presentation, initiation and activation of immune cells, transport and infiltration of immune cells into the tumor, and recognition and killing of cancer cells by $\mathrm{T}$ cells. Consequently, the cancer immunity cycle has become one of the starting points for cancer immunotherapy research. Therefore, it is imperative to study the TME and identify the distribution and functions of tumor-infiltrating immune cells (TIICs) to find new tumor markers for thyroid cancer.

SBSN was first identified in epithelial tissues (human and murine) and is thought to play a key role in the process of epidermal differentiation (Park et al., 2002). However, in recent years, SBSN has been reported to be aberrantly expressed in certain malignancies, and inhibition of SBSN may lead to the inhibition of cancer cell proliferation, invasion, and metastasis, suggesting that SBSN may be associated with tumor progression. For example, SBSN expression is abnormally regulated in esophageal squamous cell carcinoma (ESCC) (Zhu et al., 2016; Takahashi et al., 2020), salivary adenoid cystic carcinoma (ACC) (Shao et al., 2012), and NSCLC (Glazer et al., 2009). In addition, several studies have shown that SBSN expression in tumors is regulated by several signaling pathways that affect the tumor properties (Alam et al., 2014; Zhu et al., 2016; Takahashi et al., 2020). These results suggest that SBSN may act as an oncogenic factor to promote tumorigenesis and tumor progression. In addition, SBSN plays an important role in the development and progression of immune diseases, such as neuropsychiatric systemic lupus erythematosus (Ichinose et al., 2018) and atopic dermatitis (Aoshima et al., 2019). However, the potential mechanism of SBSN as a proto-oncogene in thyroid cancer progression and immunology is not clear.

In this study, we investigated the expression of SBSN in thyroid cancers of follicular epithelial origin with different degrees of differentiation. The relationship of SBSN with clinicopathological features of patients with PTC, as well as the potential involvement of SBSN in cancer immunity, were 
also explored. This study suggests an important role for SBSN in thyroid carcinoma and the potential mechanisms by which SBSN may be involved in the processes of thyroid cancer dedifferentiation and immune regulation.

\section{MATERIALS AND METHODS}

\section{Data Sources and Pre-Processing}

This study used several public datasets, including PTC, PDTC, and ATC datasets. For The Cancer Genome Atlas (TCGA) dataset, RNA sequencing (RNA-seq) data and clinical features of patients with thyroid cancer were identified in and extracted from the TCGA portal and validated (Cancer Genome Atlas Research Network, 2014), with a total of 568 samples, including 502 PTCs, 8 metastatic thyroid cancers, and 58 matched normal thyroid samples. High-throughput sequencing fragments per kilobase of transcript per million mapped reads (FPKM) values were further analyzed for all samples after $\log _{2}(\mathrm{FPKM}+1)$ transformation. The ATC microarray datasets GSE29265, GSE33630, GSE76039, and GSE65144 were downloaded from the Gene Expression Omnibus database of the National Center for Biotechnology Information by searching for "anaplastic thyroid cancer" and "Homo sapiens." The PDTC microarray datasets GSE53157 and GSE76039 were downloaded from the same database by searching for "poorly differentiated thyroid cancer" and "Homo sapiens." All microarray data were background adjusted and normalized by removing the batch processing effect using the $\mathrm{R}$ package "sva." Probes that did not match the gene symbol in the annotation file were deleted. When more than one probe matched the same gene symbol, the average value was calculated as the final expression value. GSE29265 consisted of 9 ATC tissues and 10 adjacent normal thyroid tissues. GSE33630 consisted of 11 ATC tissues and 45 adjacent normal thyroid tissues. GSE65144 consisted of 12 ATC tissues and 13 adjacent normal thyroid tissues. GSE76039 consisted of 17 PDTC tissues and 20 ATC tissues. GSE53157 consisted of five PDTCs, seven classical PTCs, eight PTC follicular variants, and four follicular thyroid carcinomas.

\section{Immune Infiltration Analysis}

The ESTIMATE algorithm can determine the ratio of stromal and immune cells based on the gene expression profile in tumor samples. It has been applied to assess the TME in patients, as well as the stromal score (stromal cell content), immune score (degree of immune cell infiltration), ESTIMATE score (a synthetic marker of the stroma and immunity), and tumor purity, using the R package (Yoshihara et al., 2013). The CIBERSORT-ABS and $\mathrm{xCell}$ algorithms were used to estimate the relative proportions of various immune cell types in the TME. For each cell type, xCell was used to analyze the enrichment scores of all samples by integrating a single-sample gene set enrichment analysis (ssGSEA) approach (Aran et al., 2017). CIBERSORT-ABS, an analytical method developed by Newman, uses gene expression data to estimate the abundance ratios of 22 cell types in a mixed cell population at a statistical significance level of $p<0.05$ (Newman et al., 2015). The reference for the deconvolution of
RNA-seq data from patients with cancer was the leukocyte signature matrix (LM22). For cancer immunity cycle analysis, we applied the Tracking Tumor Immunophenotype (TIP) pipeline based on the ssGSEA algorithm (Xu et al., 2018). The TIP scores for the TCGA dataset are available from the online TIP server (http://biocc.hrbmu.edu.cn/TIP/).

\section{Patients and Clinicopathological Data}

Eighty specimens from patients with PTC who underwent surgical treatment at the Shengjing Hospital of China Medical University from July 2016 to July 2017 were stored in liquid nitrogen and subjected to quantitative reversetranscription polymerase chain reaction (qRT-PCR) and western blotting. For immunohistochemical staining, tissues were embedded in paraffin. The selected PTC tissues and paired normal tissues adjacent to cancer were diagnosed by pathology. Normal tissues adjacent to the cancer tissue were collected, from the same patients with PTC, at least $2 \mathrm{~cm}$ from the PTC area. Paraffin-embedded tissues from 9 patients with PDTC and 18 patients with ATC who underwent surgical treatment at the Shengjing Hospital of China Medical University from 2010 to 2017 were preserved at the Department of Pathology. All histological sections were reviewed by two specialist pathologists to verify the histological diagnosis. All patients were diagnosed for the first time and did not receive any treatment before surgery. Patients were classified according to the eighth edition of the American Joint Committee on Cancer TNM classification system for differentiated thyroid cancer. Clinical information such as patient age, tumor size, and cervicallymph-node metastasis was retrieved from the clinical files of the patients. All patients provided informed consent for the use of their clinical and pathological data for research purposes, and all tissue specimens and clinical data were collected according to the protocol approved by the Ethics Committee of the Shengjing Hospital, China Medical University (approval number 2014PS47K).

\section{Functional and Pathway Enrichment Analyses}

The GeneMANIA (http://www.genemania.org) database was used to construct a gene-gene interaction network for $S B S N$, including genes that are associated with SBSN in terms of physical interactions, co-expression, prediction, colocalization, and genetic interactions. Functional and pathway enrichment analyses of the genes co-expressed with SBSN in the cBioPortal database (496 cases from the TCGA Cell 2014 dataset of PTC) were performed using DAVID (https://david.ncifcrf.gov). Genome enrichment analysis was performed using GSEA 3.0 software. The c2.cp.kegg.v6.1. symbols.gmt dataset was downloaded from the Molecular Signatures Database on the GSEA website. Enrichment analysis of $S B S N^{\text {high }}$ and $S B S N^{\text {low }}$ groups was performed for expression spectrum data and attribute files using the default weighted method. The random classification frequency was set to 1,000 . 


\section{Immunohistochemical Staining}

Formalin-fixed, paraffin-embedded sections ( $4 \mu \mathrm{m}$ thick) were prepared and subjected to ethanol gradient dewaxing and endogenous peroxidase blocking. Antigen retrieval was performed by boiling the slides in citrate buffer ( $\mathrm{pH}$ 6.0) for $7.5 \mathrm{~min}$, followed by cooling to room temperature. The slides were incubated with a polyclonal rabbit anti-human SBSN antibody (1:250; Cat\# abx130453; Abbexa, Cambridge, United Kingdom) at $4^{\circ} \mathrm{C}$ overnight after $30 \mathrm{~min}$ of incubation at $37^{\circ} \mathrm{C}$ with drops of goat blocking serum. Afterward, the slides were rinsed with phosphate-buffered saline (PBS) and incubated with a drop of a horseradish peroxidase-labeled sheep anti-rabbit secondary antibody for $30 \mathrm{~min}$ at $37^{\circ} \mathrm{C}$. Subsequently, the slides were stained with a 3,3'-diaminobenzidine (Cat\# DAB-0031; MXB, Maixin, China) solution for 1-2 min, counterstained with hematoxylin, dehydrated, covered with coverslips, and analyzed by light microscopy. PBS was used instead of the primary antibody in a negative control group. The experimental procedure was carried out according to the SP kit instructions. Five high-magnification fields $(\times 400)$ were randomly selected from each section under a light microscope and scored by two pathologists. The degree of staining was scored from 0 to 4 ( 0 , none; $1,<10 \% ; 2,10-50 \% ; 3,51-80 \%$; and 4 , $>80 \%$ ). The staining intensity was scored from 0 to 3 ( 0 , no staining; 1, light yellow; 2, brown-yellow; and 3, brown). To calculate the final score, the two scores were multiplied, and the results were presented as follows: $0-1$ point $(-), 2-4$ points $(+)$, 5-8 points $(++)$, and $9-12$ points $(+++)$. We defined $-/+$ as the low-positive expression group and $++/+++$ as the high-positive expression group. To control for errors, the scoring was performed by two independent observers, and a third observer read the films; in cases of disagreement, all three discussed the scores collectively until an agreement was reached.

For M2 macrophages, fields of view with $\mathrm{CD}_{163}{ }^{+} \mathrm{M} 2$ macrophages were selected. The number of $\mathrm{CD}_{163}{ }^{+} \mathrm{M} 2$ macrophages was counted in five randomized highmagnification fields $(\times 400)$ per sample, and the mean value was considered as the level of $\mathrm{CD}_{163^{+}} \mathrm{M} 2$ macrophages.

For Tregs, fields of view with Foxp $3^{+}$Tregs were selected. The number of Foxp $3^{+}$Tregs was counted in five randomized highmagnification fields $(\times 400)$ per sample, and the mean value was considered as the level of Foxp $3^{+}$Tregs.

\section{Western Blotting}

To extract total proteins, RIPA lysis buffer (Cat\#P0013B; Beyotime Biotechnology, Shanghai, China) was added to tissues, and the homogenates were centrifuged at $14,000 \times$ $\mathrm{rpm}$ for $45 \mathrm{~min}$ at $4^{\circ} \mathrm{C}$. Total proteins $(40 \mu \mathrm{g})$ were separated by SDS-PAGE and transferred to PVDF membranes. The membranes were blocked with $5 \%$ bovine serum albumin at room temperature for $2 \mathrm{~h}$ and then incubated with primary antibodies at $4^{\circ} \mathrm{C}$ overnight. Thereafter, the membrane was incubated with a secondary antibody for $2 \mathrm{~h}$ at room temperature. The primary antibodies used in this study included a rabbit anti-SBSN polyclonal antibody (1:1,000; Cat\# abx130453; Abbexa, Cambridge, United Kingdom) and rabbit polyclonal anti-GAPDH (1:10,000; Cat\# 10494-1-AP,
Proteintech Group, Inc., Chicago, United States). Peroxidaselabeled goat anti-rabbit or anti-mouse $\operatorname{IgG}(\mathrm{H}+\mathrm{L})(1: 2,000$; Zhongshan Jinqiao Company, Beijing, China) was used as a secondary antibody. An enhanced chemiluminescence kit (Beyotime Biotechnology) was used for detection. The integrated optical density of each band was measured using Image-Pro Plus 6.0 software (Media Cybernetics Inc., Rockville, MD, United States). The target protein expression level was calculated relative to that of GAPDH, which was used as an internal control.

\section{qRT-PCR}

The total RNA was extracted from thyroid tissue specimens using TRIzol reagent (Cat\# 9108; Takara, Beijing, China) according to the manufacturer's instructions. After verification of the purity and concentration, RNA was reverse transcribed into cDNA using a cDNA synthesis kit (Cat\# RR047A; Takara, Beijing, China). qRT-PCR of the cDNA ( $2 \mu \mathrm{l}$ per $20 \mu$ reaction) was performed using the TB Green ${ }^{\circledR}$ Premix Ex Taq TM II kit (Cat\# RR820; Takara, Beijing, China) and a 7,500 Fast instrument. Primer sequences for SBSN were forwad:5' CATGGCGTTAGTCAGGCTGGAAG-3' and reverse: $5^{\prime}$ CCTCCTTGCTGGCTTGGTTGAC- $3^{\prime}$. The primer sequences used for GAPDH were forward:5' GGAGCGAGATCCCTCCAAAAT- $3^{\prime}$ and reverse:5' - GGC TGTTGTCATACTTCTCATGG-3'. The PCR protocol was as follows: $95^{\circ} \mathrm{C}$ for $2 \mathrm{~min}$, followed by 40 cycles at $95^{\circ} \mathrm{C}$ for $15 \mathrm{~s}$ and $60^{\circ} \mathrm{C}$ for $30 \mathrm{~s}$. The relative expression level was calculated using the $2^{-\triangle \Delta C t}$ method using $G A P D H$ as a reference gene for normalization.

\section{Statistical Analysis}

Statistical analyses were performed using SPSS (20.0) and R (4.0.3) software. The chi-squared test was used to analyze differences in the degree of SBSN immunohistochemical staining among ATC, PDTC, PTC, and adjacent normal thyroid tissues. The relationships between the statistical results of SBSN immunohistochemical staining and the clinicopathological characteristics of PTC were assessed by the chi-squared test and Fisher's exact probability test. A $t$-test was used to assess the relationships between the statistical results of SBSN western blotting and qRT-PCR and the clinicopathological characteristics of PTC. Data are expressed as means \pm standard deviation. Box plot analysis was performed using the Wilcoxon rank-sum test; correlation between two variables was calculated using Spearman's rho, and one-way analysis of variance was used for comparison among multiple samples. $p<0.05$ was considered to be statistically significant.

\section{RESULTS}

\section{Expression of SBSN in Thyroid Cancer Tissues}

The relative expression level of SBSN mRNA was evaluated in the 80 PTC tissues and paired paraneoplastic normal tissues. The qRT-PCR results showed that the relative expression level of 
A

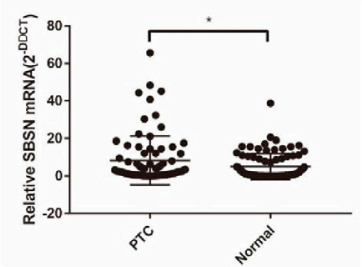

D

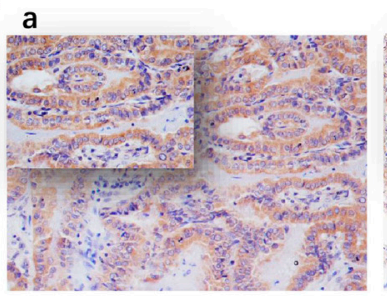

d

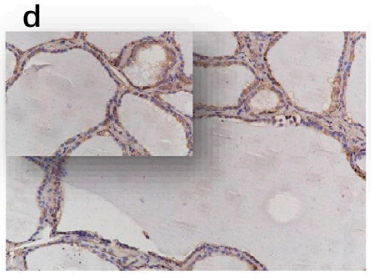

B

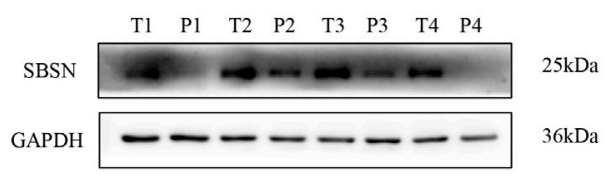

b

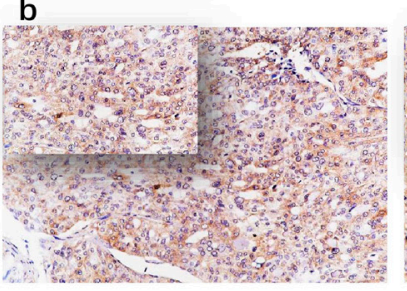

c

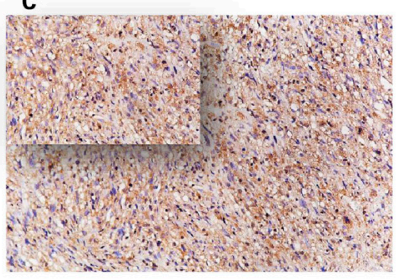

C

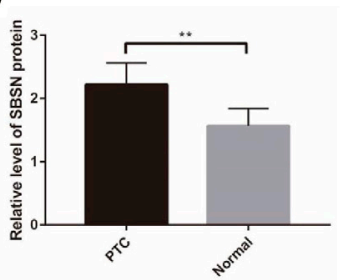

E

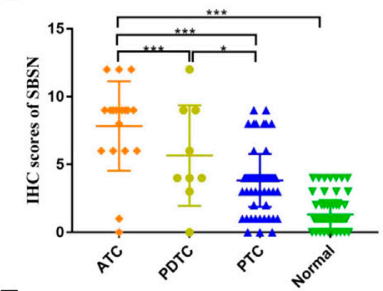

F
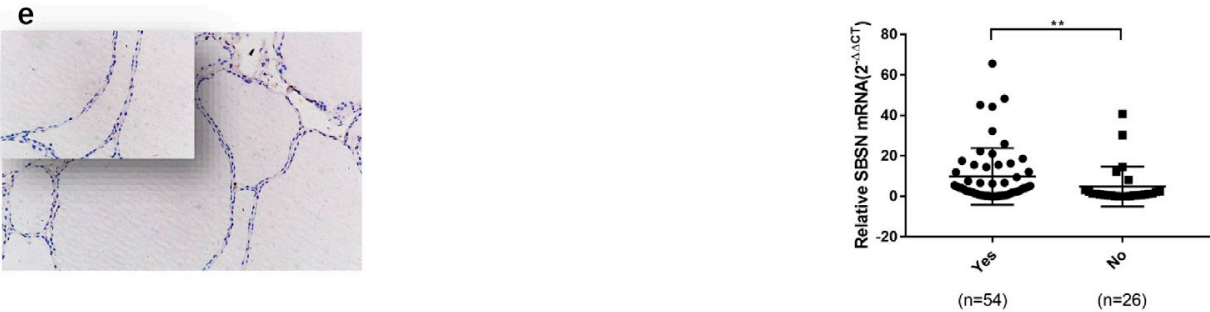

FIGURE 1 | Expression of SBSN in different thyroid tissues. (A) Expression of SBSN in papillary thyroid carcinoma (PTC) tissues and normal tissues adjacent to cancer was detected by qRT-PCR ( $n=80$ per group). (B) Expression of SBSN in PTC and normal tissues adjacent to cancer was detected using western blot $(n=80$ per group). (C) Relative grayscale values of SBSN in PTC tissues and normal tissues adjacent to cancer. (D) Expression of SBSN in different thyroid cancer tissue samples ( $\times 200$, top left $\times 400$ ). (a) Positive expression of SBSN in PTC tissues; (b) Positive expression of SBSN in poorly differentiated thyroid carcinoma tissues; (c) Positive expression of SBSN in anaplastic thyroid carcinoma tissues; (d) Positive expression of SBSN in normal tissues adjacent to cancer; (e) Negative expression of SBSN in normal tissues adjacent to cancer. (E) Immunohistochemical staining scores of SBSN in various thyroid tissue samples. (F) High expression of SBSN mRNA levels in PTC tissues was associated with lymph node metastasis in patients. For western blot, GAPDH was used as an internal control. Data are expressed as means \pm standard deviation. ${ }^{*} p<0.05,{ }^{* *} p<0.01,{ }^{* \star} p<0.001$.

TABLE 1 | Expression of SBSN in different types of thyroid tissue.

\begin{tabular}{|c|c|c|c|c|c|c|}
\hline \multirow[t]{2}{*}{ Group } & \multirow[t]{2}{*}{$n$} & \multicolumn{2}{|c|}{ Low } & \multicolumn{2}{|c|}{ High } & \multirow{2}{*}{$\begin{array}{c}\text { High positive rate } \\
\text { (\%) }\end{array}$} \\
\hline & & $(-)$ & (+) & $(++)$ & $(+++)$ & \\
\hline ATC & 18 & 2 & 0 & 5 & 11 & $88.89^{a, b}$ \\
\hline PDTC & 9 & 1 & 4 & 1 & 3 & $44.44^{c}$ \\
\hline PTC & 80 & 12 & 58 & 8 & 2 & $12.5^{d}$ \\
\hline Normal & 80 & 61 & 19 & 0 & 0 & 0 \\
\hline
\end{tabular}

${ }^{a} A T C$, vs. PDTC $\left({ }^{*} \mathrm{p}=0.023\right)$.

${ }^{b}$ ATC, vs. PTC $(* * * 00.001)$.

${ }^{c}$ PDTC, vs. PTC $\left({ }^{*} \mathrm{p}=0.044\right)$.

${ }^{d} P T C$, vs Normal $\left.{ }^{* * *} p=0.001\right)$.

SBSN mRNA was significantly higher in the PTC tissues than that in the paraneoplastic normal tissues $(8.228 \pm 1.452$ and $5.037 \pm$ .783 , respectively; $p<0.05$ ) (Figure 1A). Western blot results showed that the expression level of SBSN in the 80 PTC tissues was significantly higher than that in the paraneoplastic tissues $(2.218 \pm .343$ and $1.563 \pm .279$, respectively; $p<0.05)$ (Figures 1B,C). Immunohistochemical staining showed that SBSN protein was mainly expressed in the cytoplasm (Figure 1D). The high- positive expression rate of SBSN was $12.5 \%(10 / 80)$ in the PTC tissues and $0 \%(0 / 80)$ in the adjacent normal tissues $(p=.001)$. In addition, the expression of SBSN in the 9 patients with PDTC and 18 patients with ATC was detected by immunohistochemical staining. The results showed that the high-positive expression rate of SBSN in ATC $(88.9 \%, 16 / 18)$ was significantly higher $(p<$ $0.05)$ than those in PDTC $(44.4 \%, 4 / 9)$ and PTC $(12.5 \%, 10 / 80)$. Compared with that in PTC, the high-positive expression rate of SBSN was significantly higher $(p<0.05)$ in the PDTC group (Table 1). The immunohistochemical staining scores are shown in Figure 1E.

\section{Relationships Between High SBSN Expression Level and Clinicopathological Characteristics of Patients With PTC}

We characterized the clinicopathological characteristics, including the expression levels of SBSN, in 80 PTC patients. As shown in Table 2, the expression levels of SBSN mRNA were significantly different between the groups with and without lymph node metastasis $(9.84 \pm 1.91$ and $4.87 \pm 1.93$, respectively; $p<0.05$ ) (Figure $1 \mathbf{1 F}$ ). The SBSN protein 
TABLE 2 | Relationship between SBSN and the clinical pathological characteristics in papillary thyroid carcinoma.

\begin{tabular}{|c|c|c|c|c|c|c|c|c|}
\hline \multirow[t]{2}{*}{ Clinical features } & \multirow[t]{2}{*}{$n$} & \multicolumn{2}{|c|}{ SBSN expression [cases (\%)] ${ }^{a}$} & \multirow[t]{2}{*}{$p$} & \multirow[t]{2}{*}{ SBSN mRNA } & \multirow[t]{2}{*}{$p$} & \multirow[t]{2}{*}{ SBSN protein ${ }^{b}$} & \multirow[t]{2}{*}{$p$} \\
\hline & & Low positive & High positive & & & & & \\
\hline \multicolumn{9}{|l|}{ Gender } \\
\hline Female & 64 & 57 (89.06) & $7(10.94)$ & 0.673 & $6.62 \pm 1.25$ & 0.104 & $2.27 \pm 0.39$ & 0.338 \\
\hline \multicolumn{9}{|l|}{ Age(y) } \\
\hline$\geq 55$ & 23 & $20(86.96)$ & $3(13.04)$ & 0.926 & $8.59 \pm 3.26$ & 0.889 & $2.86 \pm 0.91$ & 0.301 \\
\hline$\leq 2$ & 58 & $53(91.38)$ & $5(8.62)$ & \multirow[t]{3}{*}{0.150} & $7.82 \pm 1.75$ & \multirow[t]{3}{*}{0.949} & $1.86 \pm 0.36$ & \multirow[t]{3}{*}{0.054} \\
\hline$>2, \leq 4$ & 20 & $15(75)$ & $5(25)$ & & $9.69 \pm 2.84$ & & $2.61 \pm 0.76$ & \\
\hline$>4$ & 2 & $2(100)$ & $0(0)$ & & $2.63 \pm 0.10$ & & $6.71 \pm 2.80$ & \\
\hline \multicolumn{9}{|l|}{ Multifocality } \\
\hline Yes & 43 & $41(95.35)$ & $2(4.65)$ & \multirow[t]{2}{*}{0.051} & $7.56 \pm 1.90$ & \multirow[t]{2}{*}{0.758} & $2.22 \pm 0.47$ & \multirow[t]{2}{*}{0.679} \\
\hline No & 37 & $29(78.38)$ & $8(21.62)$ & & $8.93 \pm 2.23$ & & $2.21 \pm 0.51$ & \\
\hline \multicolumn{9}{|l|}{ Bilateral } \\
\hline No & 72 & 64 (88.89) & $8(11.11)$ & 0.573 & $8.13 \pm 1.55$ & & $2.00 \pm 0.30$ & \\
\hline Lymph node metas & & & & & & & & \\
\hline Yes & 54 & $44(81.48)$ & 10 (18.52) & 0.047 & $9.84 \pm 1.91$ & 0.010 & $2.71 \pm 0.49$ & 0.042 \\
\hline No & 26 & $26(100)$ & $0(0)$ & & $4.87 \pm 1.93$ & & $1.19 \pm 0.20$ & \\
\hline ACJJ stage & & & & & & & & \\
\hline I-II & 74 & 65 (87.84) & $9(12.16)$ & 0.564 & $8.24 \pm 1.50$ & 0.811 & $2.25 \pm 0.36$ & 0.771 \\
\hline III-IV & 6 & 5 (83.33) & $1(16.67)$ & & $7.99 \pm 4.69$ & & $1.50 \pm 0.43$ & \\
\hline Tumor grade & & & & & & & & \\
\hline G1 & 26 & $23(88.46)$ & $3(13.04)$ & 0.857 & $10.26 \pm 3.22$ & 0.486 & $1.98 \pm 0.49$ & 0.731 \\
\hline G2 & 54 & 47 (87.04) & 7 (12.96) & & $7.72 \pm 1.63$ & & $2.28 \pm 0.41$ & \\
\hline
\end{tabular}

ammunohistochemical staining.

${ }^{b}$ Western blot.

expression level $(2.71 \pm .49$ vs. $1.19 \pm .20)$ and high-positive expression rate $(18.52$ vs. $0 \%)$ were significantly higher $(p<.05)$ in the lymph node metastasis group than in the non-metastasis group, respectively. Other clinicopathological characteristics, such as age, sex, multifocality of PTC, extra-envelope infiltration, tumor size, and clinicopathological stage and grade, were not associated with the relative expression level of SBSN ( $p>.05$; Table 2). The data suggest that SBSN can influence the malignancy of PTC, thus promoting lymph node metastasis. In addition, we analyzed the relationship between SBSN expression and prognosis in 502 PTC patients with complete clinical information in conjunction with the TCGA database and found that SBSN expression had no effect on the survival time of patients (Supplementary Figure S1A), which may be attributed to the good prognosis of PTC and the low number of deaths during follow-up (16/502).

\section{Biological Interaction Networks of SBSN}

We identified the top 20 genes associated with SBSN through the GeneMANIA website, which showed SBSN as the central node surrounded by 20 other nodes (Figure 2A). The five most relevant genes included PTEN-inducible putative kinase 1 (PINK1), tankyrase (TNKS), HAUS augmin-like complex subunit 1 (HAUS1), DNA polymerase kappa (POLK), and glycogen synthase kinase $3 \beta$ (GSK3B), all of which physically interacted with $S B S N$. Further functional analysis showed that these genes were associated with protein hydrolysis, amino acid modification, cell adhesion, and regulation of apoptosis. In addition, using the DAVID tool and the "ggplot2" R package, we performed functional and pathway enrichment analyses of genes in the cBioPortal database that were co-expressed with SBSN to view the biological functions and pathways associated with SBSN. Gene Ontology enrichment analysis revealed that $S B S N$ co-expressed genes were associated with a variety of processes, including regulation of signaling, regulation of cellular signal transduction and communication, and cellular responses to chemical stimuli. They were also associated with cellular components, including the neuronal fraction and plasma membrane, and molecular functions, such as enzyme binding, activation of nucleic acid binding transcription factors, and binding of some ions (Figure 2B). Meanwhile, the enrichment of Kyoto Encyclopedia of Genes and Genomes pathways of SBSN-associated genes suggested that SBSN was associated with the $\mathrm{PI} 3 \mathrm{~K} / \mathrm{AKT}$, mitogen-activated protein kinase (MAPK), and other pathways commonly found in tumors. Interestingly, the cytokine receptor interaction pathway, chemokine signaling pathway, and T-cell receptor signaling pathway, which are associated with tumor immunity, were also enriched in SBSN co-expressed genes (Figure 2C). Similar results were obtained by ssGSEA of the published thyroid dataset (TCGA), wherein samples with high SBSN expression levels were associated with levels of cytokines 


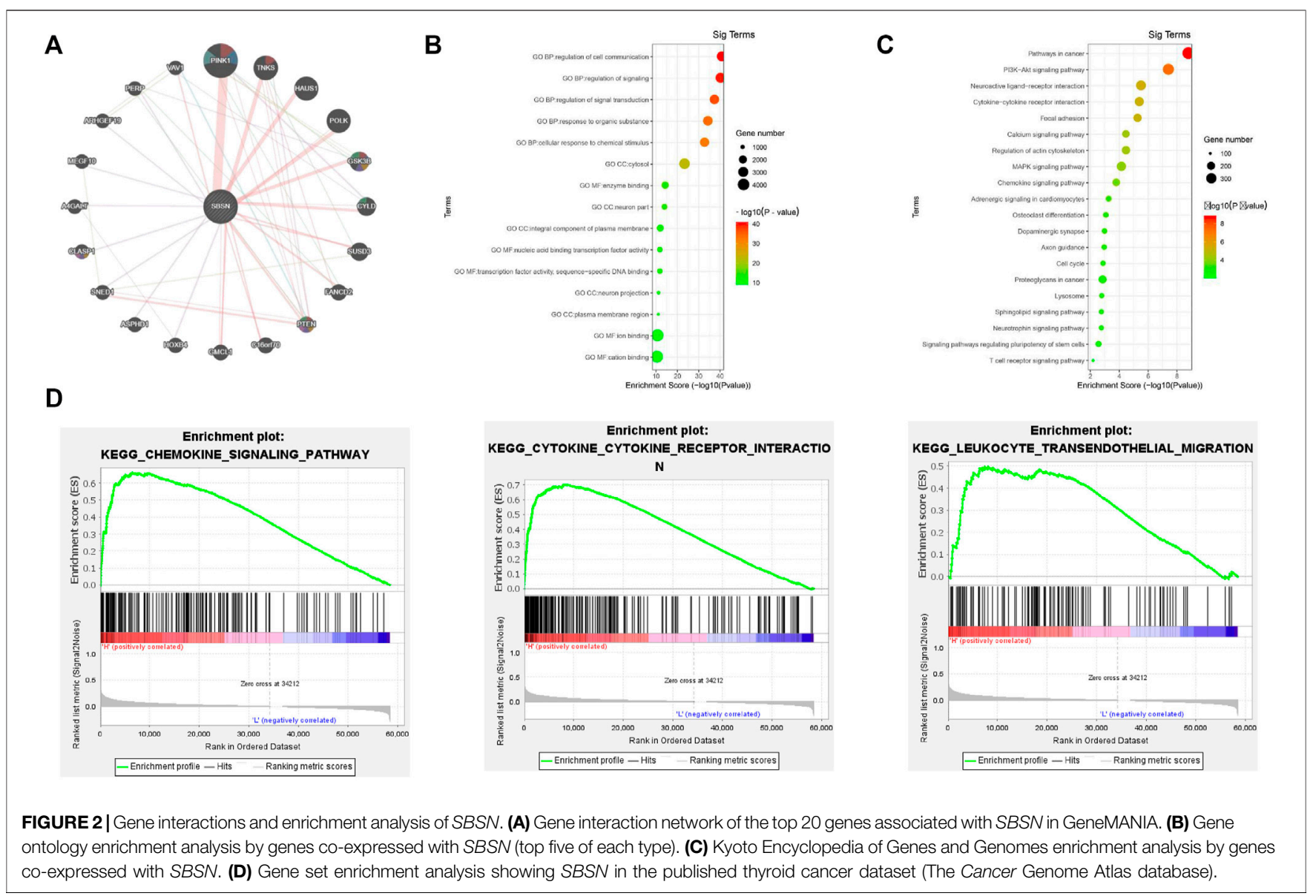

and chemokines and more pronounced leukocyte migration (Figure 2D).

\section{Correlation of SBSN Expression Levels With Immune and Stromal Cells in the TME}

To assess the distribution of immune and stromal cells in PTC, PDTC, and ATC samples, we analyzed the 502 primary PTC samples from the TCGA dataset; 52 ATC samples from GSE29265, GSE33630, GSE76039, and GSE65144; and 22 PDTC samples from GSE53157 and GSE76039. Based on the median values of SBSN expression levels in the TCGA PTC cohort, PDTC joint cohort, and ATC joint cohort, the samples were divided into $S B S N^{\text {high }}$ and $S B S N^{\text {low }}$ groups. Based on the ESTIMATE algorithm, we found that, in the PTC samples, the immune score was higher in the $S B S N^{\text {high }}$ group than in the $S B S N^{\text {low }}$ group $(p<0.01)$, while $S B S N$ expression level was negatively correlated with tumor purity $(p<0.01)$. There was no significant association between the stromal scores and SBSN expression levels (Figure 3A). In the ATC samples, the immune and stromal scores were higher in the $S B S N^{\text {high }}$ group than in the $S B S N^{\text {low }}$ group $(p<0.05)$, and $S B S N$ expression level was negatively correlated with the extent of tumor purity $(p<0.05$; Figure 3B). In the PDTC samples, no significant associations were found between SBSN expression levels and the immune scores, stromal scores, or degree of tumor purity (Supplementary Figure S1B), which may have been due to the small sample size. In conclusion, these findings suggest that SBSN expression may impact immune cells in the PTC TME and immune and stromal cells in the ATC TME.

\section{Relationship Between SBSN Expression Level and Immune Cell Infiltration}

To explore the correlation between SBSN expression and the immune microenvironment, we inferred the abundances of TIICs using CIBERSORT-ABS and xCell. The association between SBSN expression and TIICs in PTC and ATC is shown in Figure 4. In the PTC samples, the proportions of TIIC types were significantly different between patients in the $S B S N^{\text {high }}$ and $S B S N^{\text {low }}$ groups (Figures $4 \mathbf{A}, \mathbf{B}$ ). Similarly, the proportions of TIICs in the ATC samples were significantly different between the $S B S N^{\text {high }}$ and $S B S N^{\text {low }}$ groups (Figures 4 C,D). In addition, we sought to determine whether the tumor immune microenvironment was different in patients with different SBSN expression levels. For the PTC samples, the results of the deconvolution algorithm CIBERSORT-ABS showed that the proportions of effector B cells $(p<0.05)$, resting $\mathrm{CD} 4^{+}$ memory T cells $(p<0.001)$, Tregs $(p<0.01)$, activated natural killer (NK) cells $(p<0.05)$, M0 macrophages $(p<0.01)$, M2 

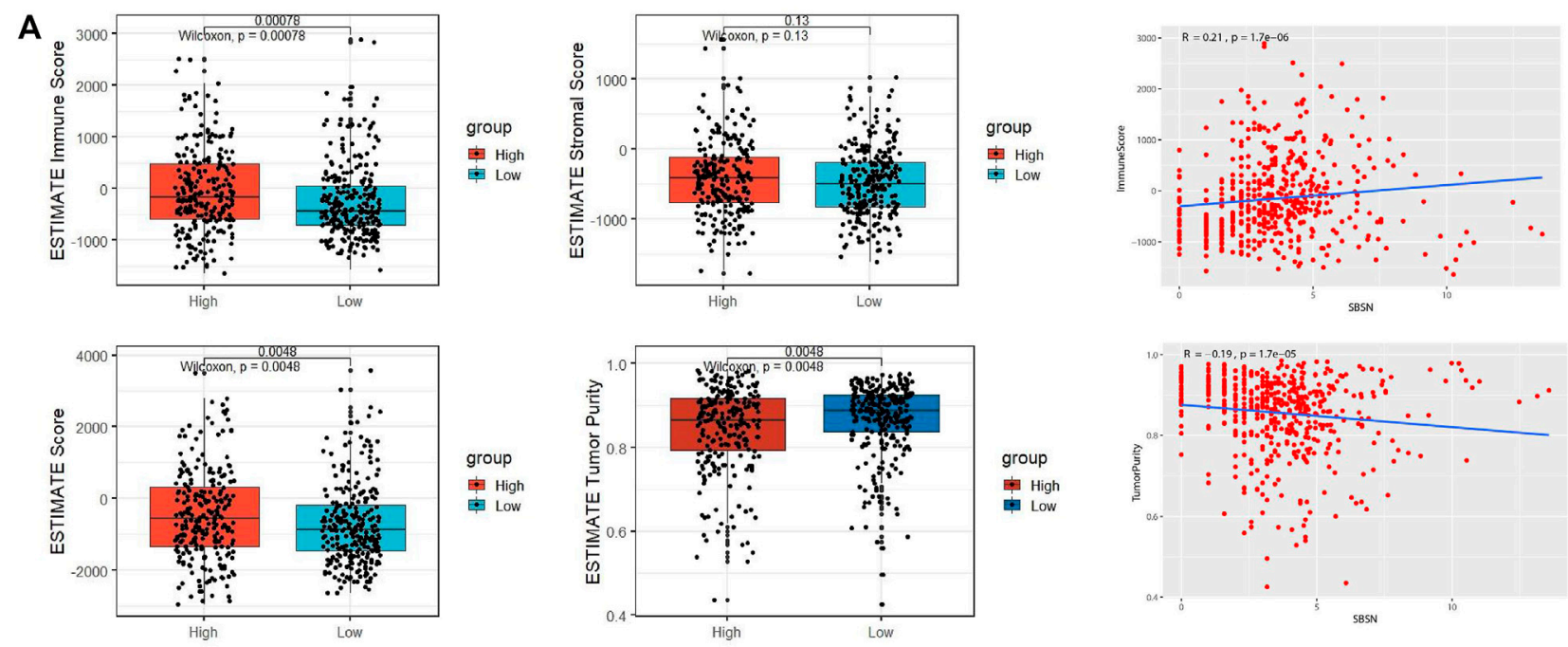

B
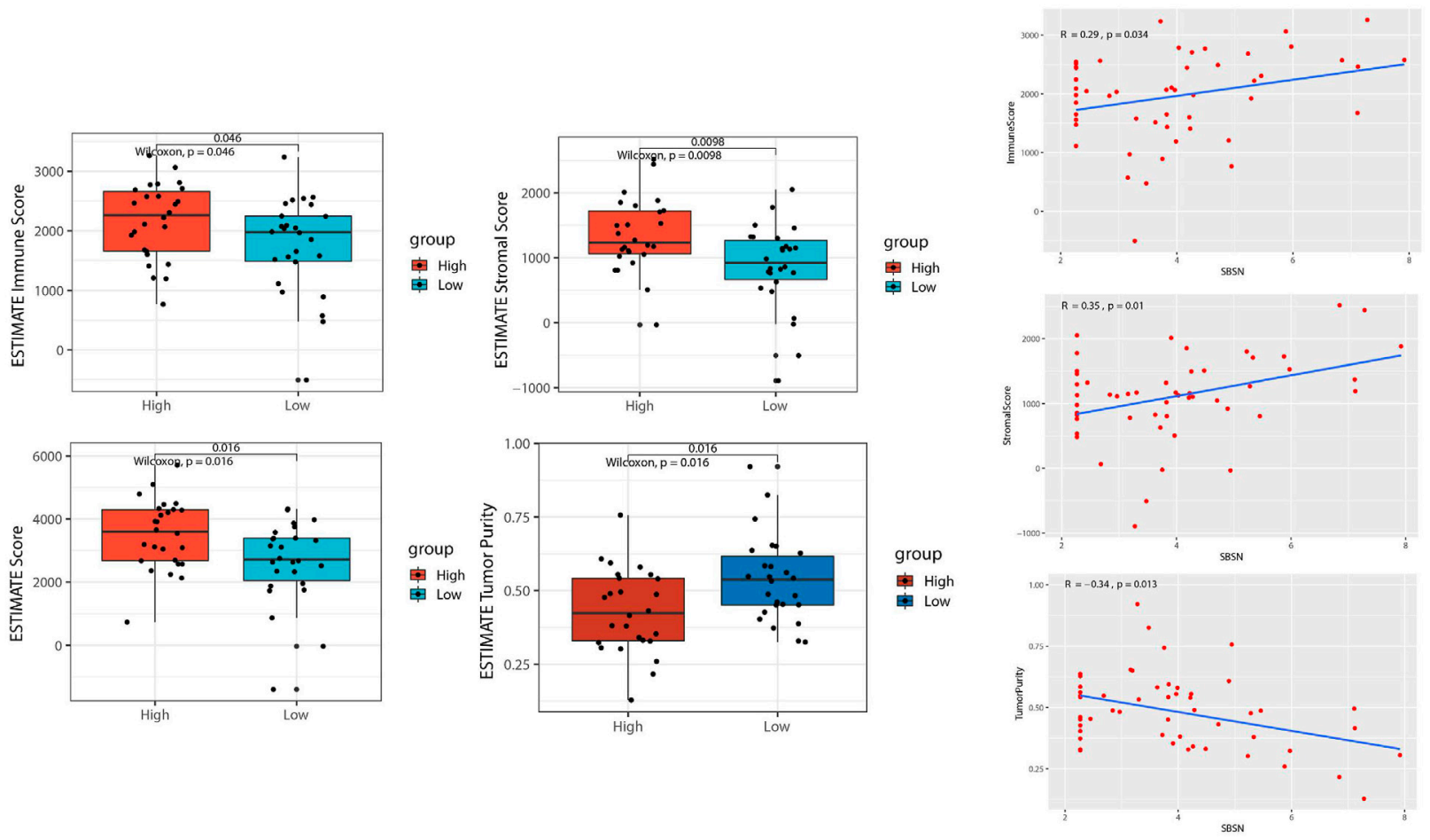

FIGURE 3 | Comparison of tumor microenvironment (TME) scores and SBSN expression profiles in follicular epithelial cell-derived thyroid cancer. (A) Comparison of the immune score, stromal score, estimate score, and tumor purity in $S B S N^{\text {high }}$ and $S B S N^{\text {low }}$ papillary thyroid carcinoma tissue groups as calculated by the ESTIMATE algorithm. (B) Comparison of the immune score, stromal score, estimate score, and tumor purity in $S B S N^{\text {high }}$ and $S B S N^{\text {low }}$ anaplastic thyroid carcinoma tissue groups as calculated by the ESTIMATE algorithm. Differences between $S B S N^{\text {high }}$ and $S B S N^{\text {low }}$ groups were compared using two-sided Wilcoxon rank-sum test.

macrophages $(p<.001)$, resting dendritic cells (DCs) $(p<.05)$, and activated DCs $(p<.0001)$ were significantly elevated in the $S B S N^{\text {high }}$ group (Figure 5A). The results of the xCell algorithm showed that 25 out of 64 noncancerous cell types were correlated and 39 cell types were not correlated with SBSN expression (Supplementary Figure S2). Among the former, 15 types had higher proportions in the $S B S N^{\text {high }}$ group, and 10 types had higher proportions in the $S B S N^{\text {low }}$ group. Numbers of activated
DCs $(p<.01)$, B cells $(p<.01)$, conventional DCs $(p<.01)$, DCs $(p<.01)$, immature DCs (iDCs) $(p<.01)$, macrophages $(p<.05)$, M2 macrophages $(p<.05)$, monocytes $(p<.01)$, and Tregs $(p<$ $.01)$ were all significantly elevated in the $S B S N^{\text {high }}$ group, while those of central memory $\mathrm{CD} 4^{+} \mathrm{T}$ cells $(p<.01)$ and $\mathrm{CD}^{+}$naïve T cells $(p<.05)$ were significantly elevated in the $S B S N^{\text {low }}$ group. In addition, some other cell types, such as keratin-forming cells $(p<.01)$, epithelial cells $(p<.01)$, platelets $(p<.05)$, astrocytes 
A

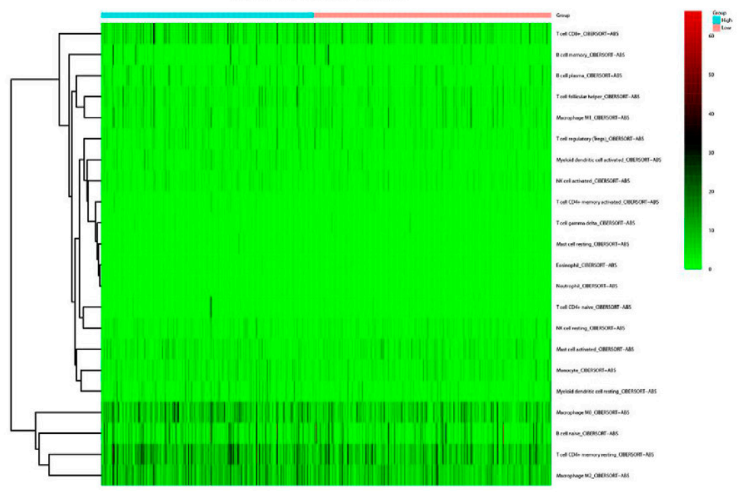

C

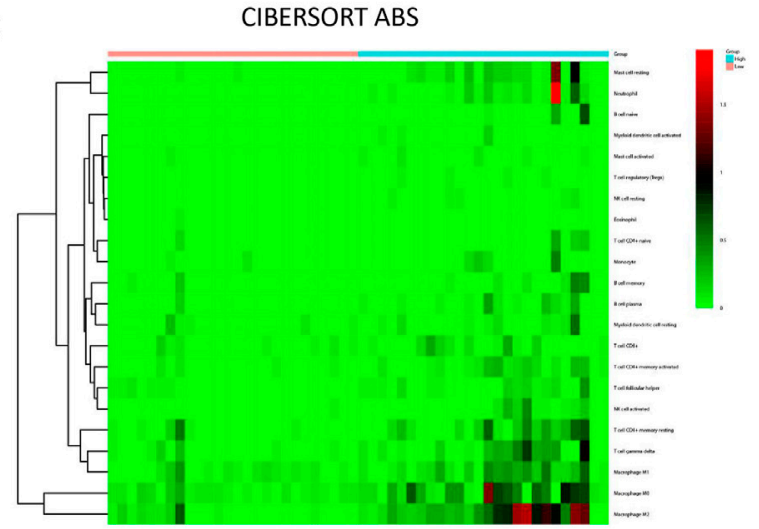

B

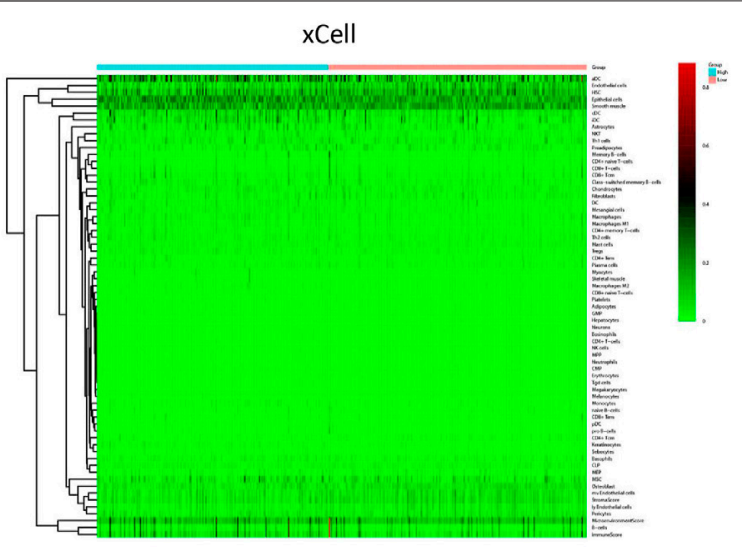

D

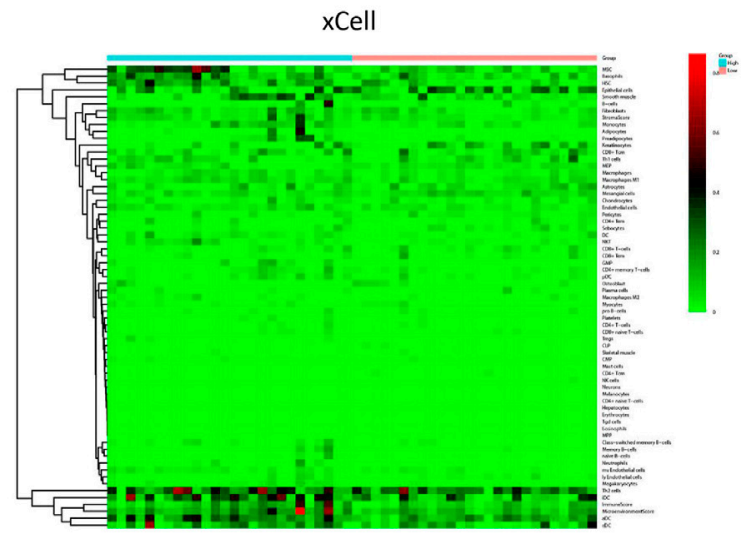

FIGURE 4 | Immune cell infiltration in patients with papillary thyroid carcinoma (PTC) and anaplastic thyroid carcinoma (ATC). (A) Heat map of tumor-infiltrating immune cell (TIIC) fractions, quantified by CIBERSORT-ABS, in PTC. (B) Heat map of TIIC fractions, quantified by xCell, in PTC. (C) Heat map of TIIC fractions, quantified by CIBERSORT-ABS, in ATC. (D) Heat map of TIIC fractions, quantified by xCell, in ATC.

$(p<.01)$, mesangial cells $(p<.01)$, and sebocytes $(p<.01)$, were more prevalent in the $S B S N^{\text {high }}$ group; meanwhile, endothelial cells $(p<.01)$, ly endothelial cells $(p<.01)$, hematopoietic stem cells (HSC) $(p<.01)$, megakaryocyte-erythroid progenitor (MEP) $(p<.01)$, multinucleated variant endothelial cells $(p<$ $.01)$, neurons $(p<.05)$, osteoblasts $(p<.01)$, and pericytes $(p<$ $.05)$ were more predominant in the $S B S N^{\text {low }}$ group (Figure 5B).

Similar to the results of ESTIMATE analysis, the CIBERSORT-ABS results showed no correlation between the immune cell content in PDTC and SBSN expression (Figure 6A), while xCell analysis yielded higher proportions of only basophils $(p<.05)$, hematopoietic stem cells $(p<.05)$, myocytes $(p<.01)$, smooth muscle cells $(p<.05)$, and T helper (Th) cell type 2 (Th2) cells $(p<.05)$ in the $S B S N^{\text {high }}$ group. The $S B S N^{\text {low }}$ group had a higher proportion of common lymphoid progenitors $(p<.05)$ as well as naïve B cells $(p<.05)$ (Figure 6B).

For the ATC samples, the CIBERSORT-ABS results showed that effector B cells $(p<.001)$, resting CD $4^{+}$memory T cells $(p<$ $.05)$, activated $\mathrm{CD} 4^{+}$memory T cells $(p<.01)$, follicular helper T cells $(p<.01)$, Tregs $(p<.05)$, resting NK cells $(p<.05)$, activated NK cells $(p<.05)$, macrophages $(p<.01)$, M1 macrophages $(p<.01)$, M2 macrophages $(p<.0001)$, resting mast cells $(p<.01)$, and neutrophils $(p<.0001)$ were significantly more numerous in the $S B S N^{\text {high }}$ group (Figure 7A). The xCell algorithm showed that 22 out of the 64 noncancerous cell types were correlated and 42 cell types were not correlated with SBSN expression (Supplementary Figure S3). Among the 22 cell types, 17 had higher proportions in the $S B S N^{\text {high }}$ group and five in the $S B S N^{\text {low }}$ group. Proportions of activated DCs $(p<.05)$, basophils $(p<.01)$, iDCs $(p<.05)$, macrophages $(p<.05)$, M2 macrophages $(p<.01)$, mast cells $(p<.01)$, monocytes $(p<.01)$, naïve B cells $(p<.05)$, and neutrophils $(p<.05)$ were significantly elevated in the $S B S N^{\text {high }}$ group. Some other cell types, such as fibroblasts $(p<$ $.05)$, granulocyte-macrophage lineage progenitors $(p<.01)$, hematopoietic stem cells $(p<.01)$, megakaryocytes $(p<.01)$, multinucleated variant endothelial cells $(p<.05)$, myocytes $(p<$ $.05)$, preadipocytes $(p<.05)$, and skeletal muscle cells $(p<.05)$ were also more common in the $S B S N^{\text {high }}$ group. Meanwhile, epithelial cells $(p<.05)$, keratin-forming cells $(p<.05)$, megakaryocyte-erythroid progenitor cells $(p<.05)$, mesangial cells $(p<.05)$, and pericytes $(p<.05)$ were more highly represented in the $S B S N^{\text {low }}$ group (Figure 7B). Taken together, 
A

A

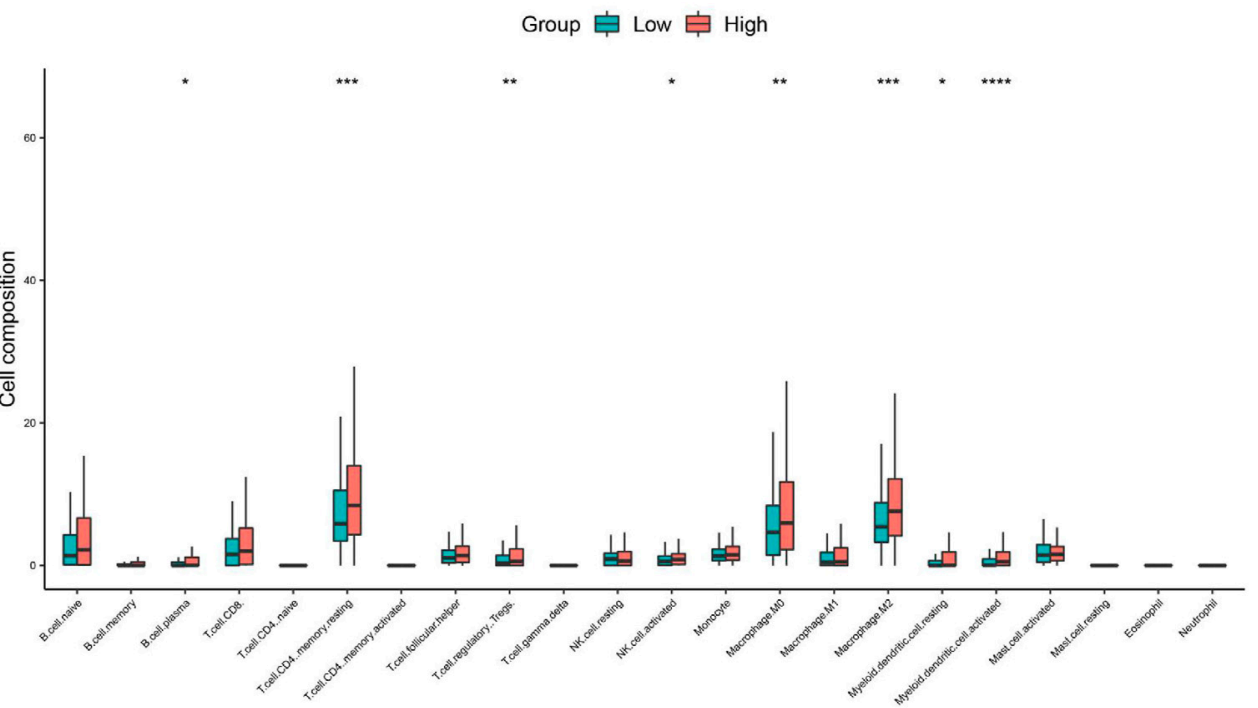

B

group 庰 High Low
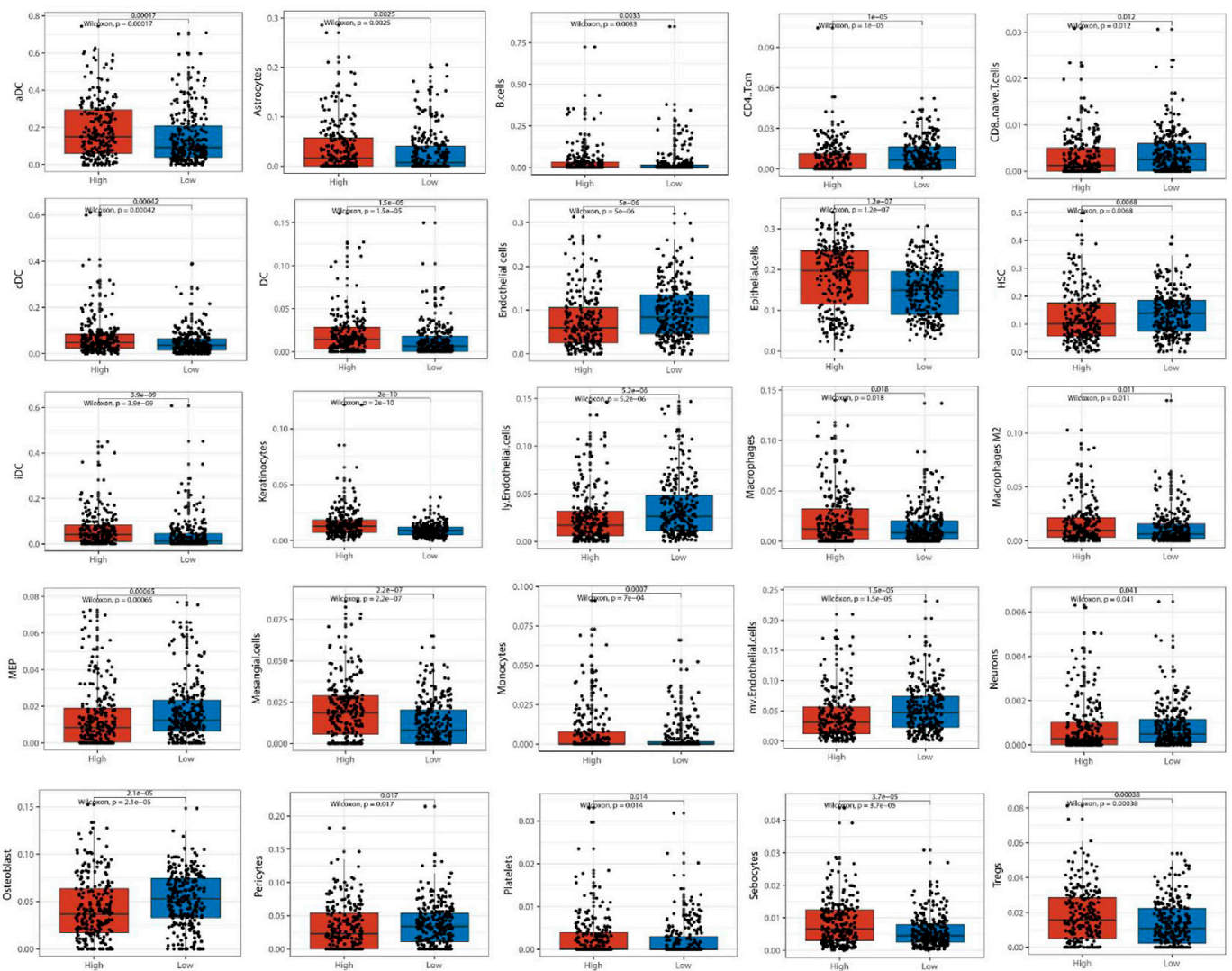

FIGURE 5 | Comparison of different non-cancer cell proportions, quantified by CIBERSORT-ABS algorithm and xCell algorithm, in SBSN ${ }^{\text {high }}$ and $S B S N^{\text {low }}$ papillary thyroid carcinoma groups. (A) Comparison of different immune cell proportions, quantified by the CIBERSORT-ABS algorithm, in the $S B S N^{\text {high }}$ and $S B S N^{\text {low }}$ groups. (B) Comparison of different non-cancer cell proportions, quantified by the $x$ Cell algorithm, in the $S B S N^{\text {high }}$ and $S B S N^{\text {low }}$ groups. Differences between $S B S N^{\text {high }}$ and $S B S N^{\text {low }}$ groups were compared using the two-sided Wilcoxon rank-sum test. ${ }^{\star} p<.05,{ }^{\star *} p<.01,{ }^{\star \star \star} p<.001,{ }^{\star \star \star *} p<.0001$. 


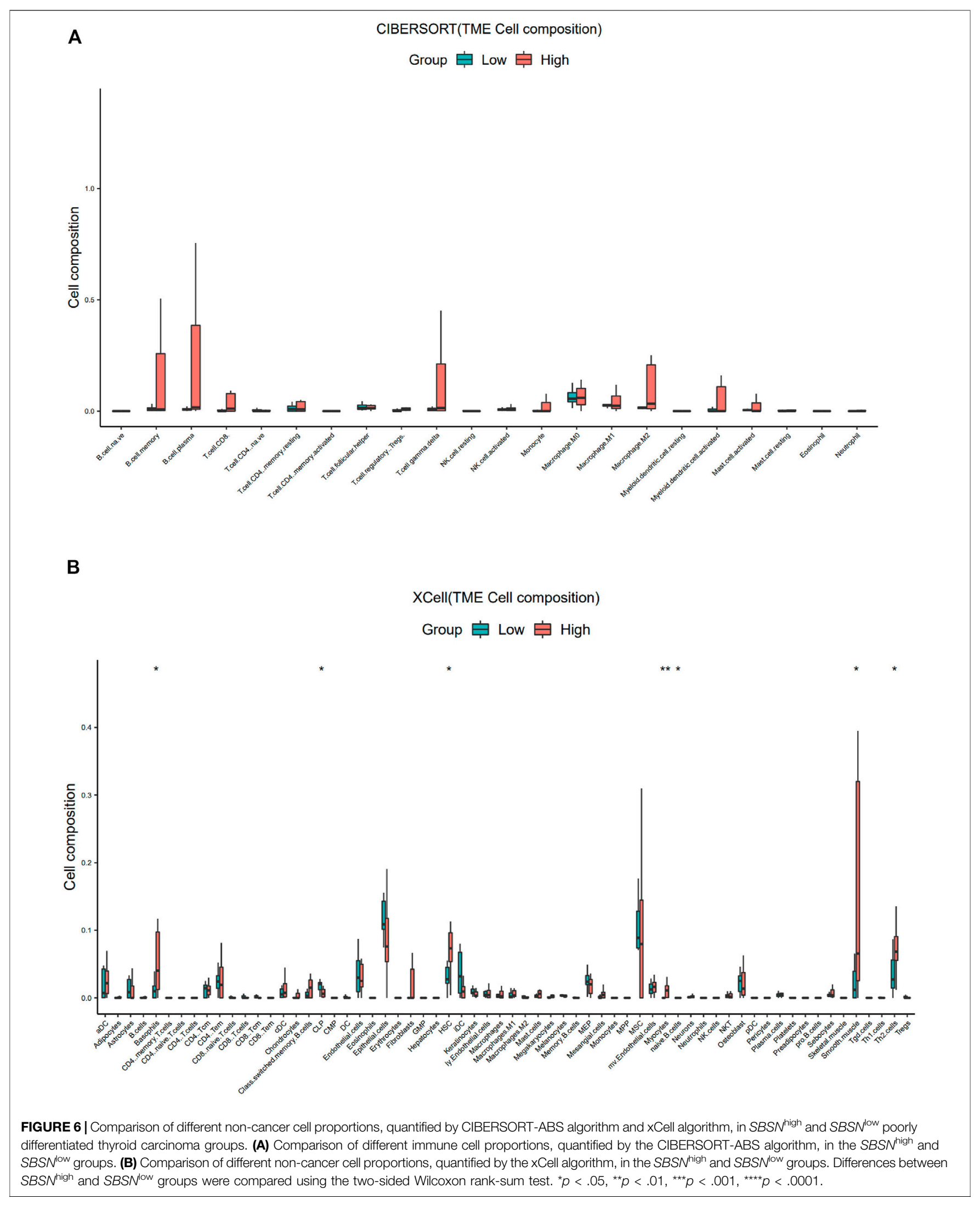




\section{A}

CIBERSORT(TME Cell composition)

Group 追 Low 户 High

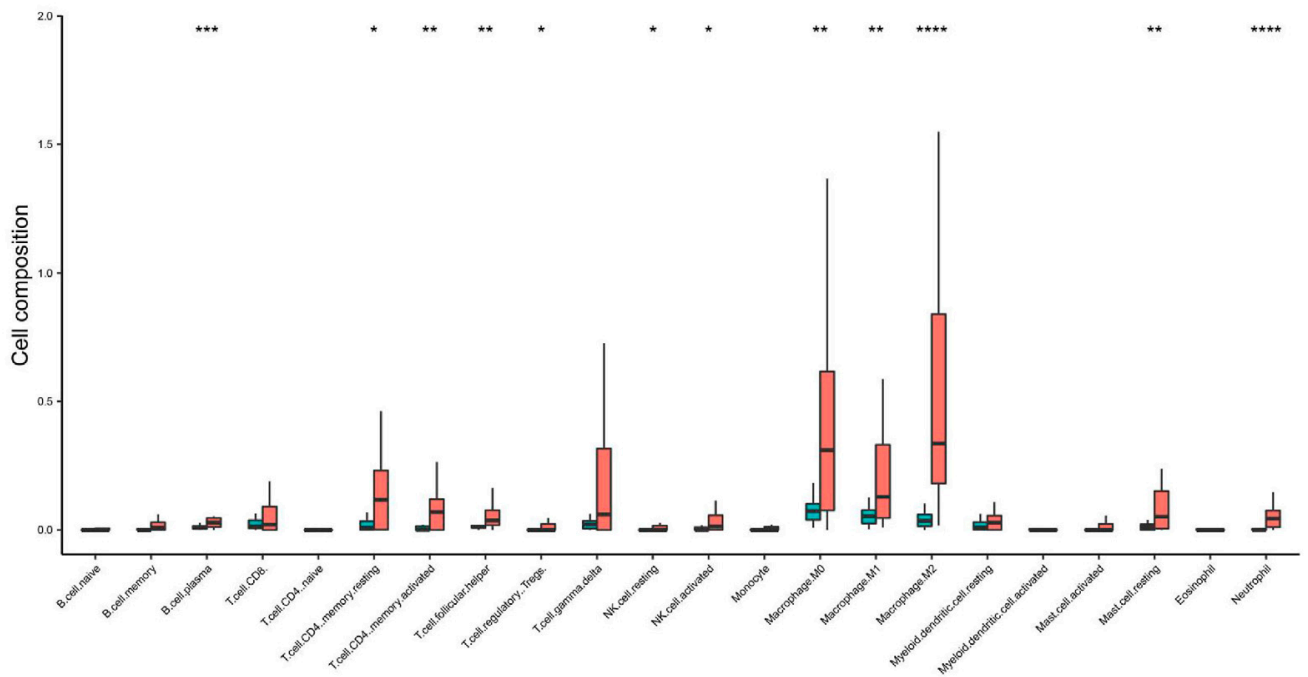

B

group 席 High Low
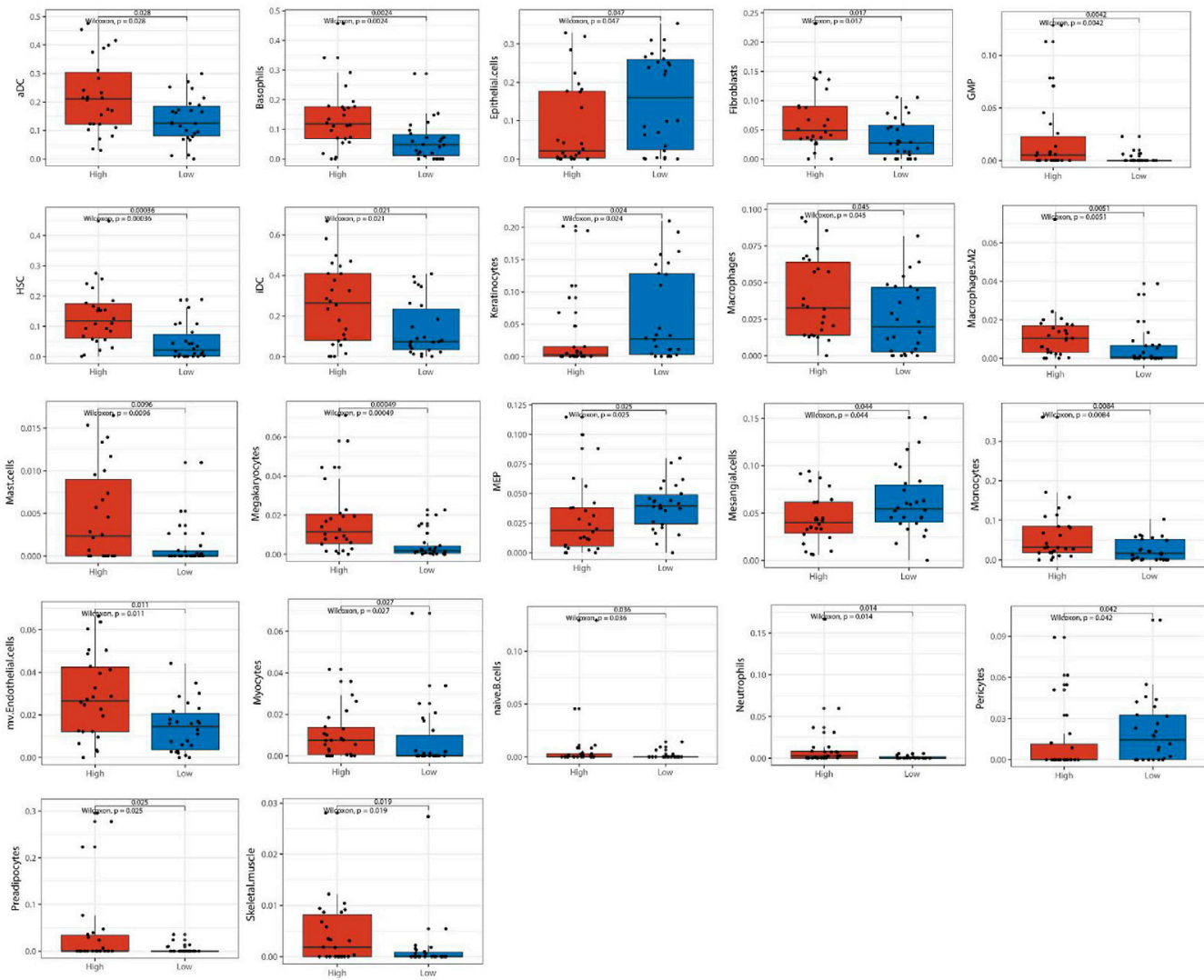

FIGURE 7 | Comparison of different non-cancer cell proportions, quantified by CIBERSORT-ABS algorithm and xCell algorithm, in SBSN ${ }^{\text {high }}$ and SBSN ${ }^{\text {low }}$ anaplastic thyroid carcinoma groups. (A) Comparison of different immune cell proportions, quantified by the CIBERSORT-ABS algorithm, in the $S B S N^{\text {high }}$ and $S B S N^{\text {low }}$ groups. (B) Comparison of different non-cancer cell proportions, quantified by the $x$ Cell algorithm, in the $S B S N^{\text {high }}$ and $S B S N^{\text {low }}$ groups. Differences between $S B S N^{\text {high }}$ and $S B S N^{\text {low }}$ groups were compared using the two-sided Wilcoxon rank-sum test. ${ }^{\star} p<.05,{ }^{\star \star} p<.01,{ }^{\star \star \star} p<.001,{ }^{\star \star \star *} p<.0001$. 


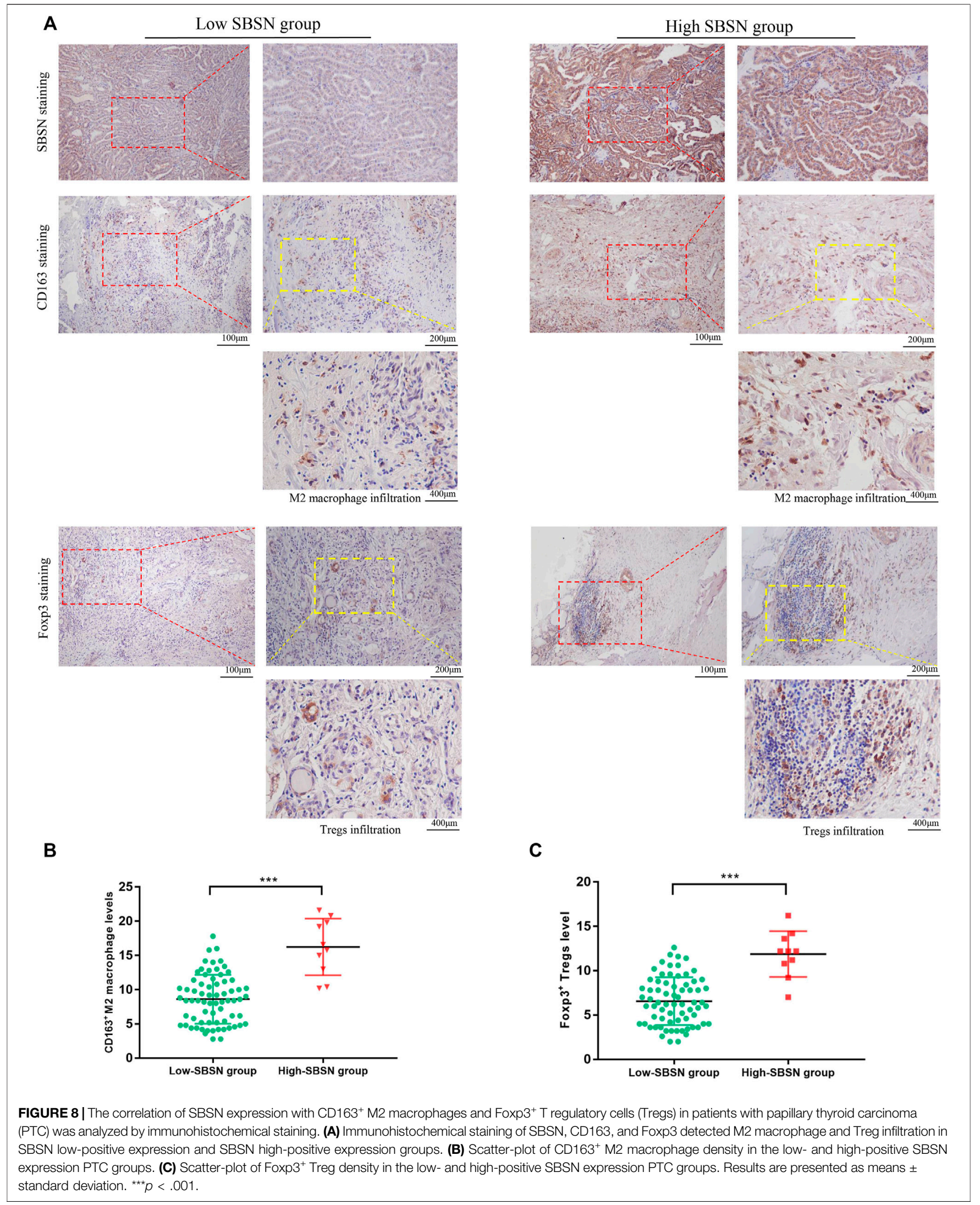




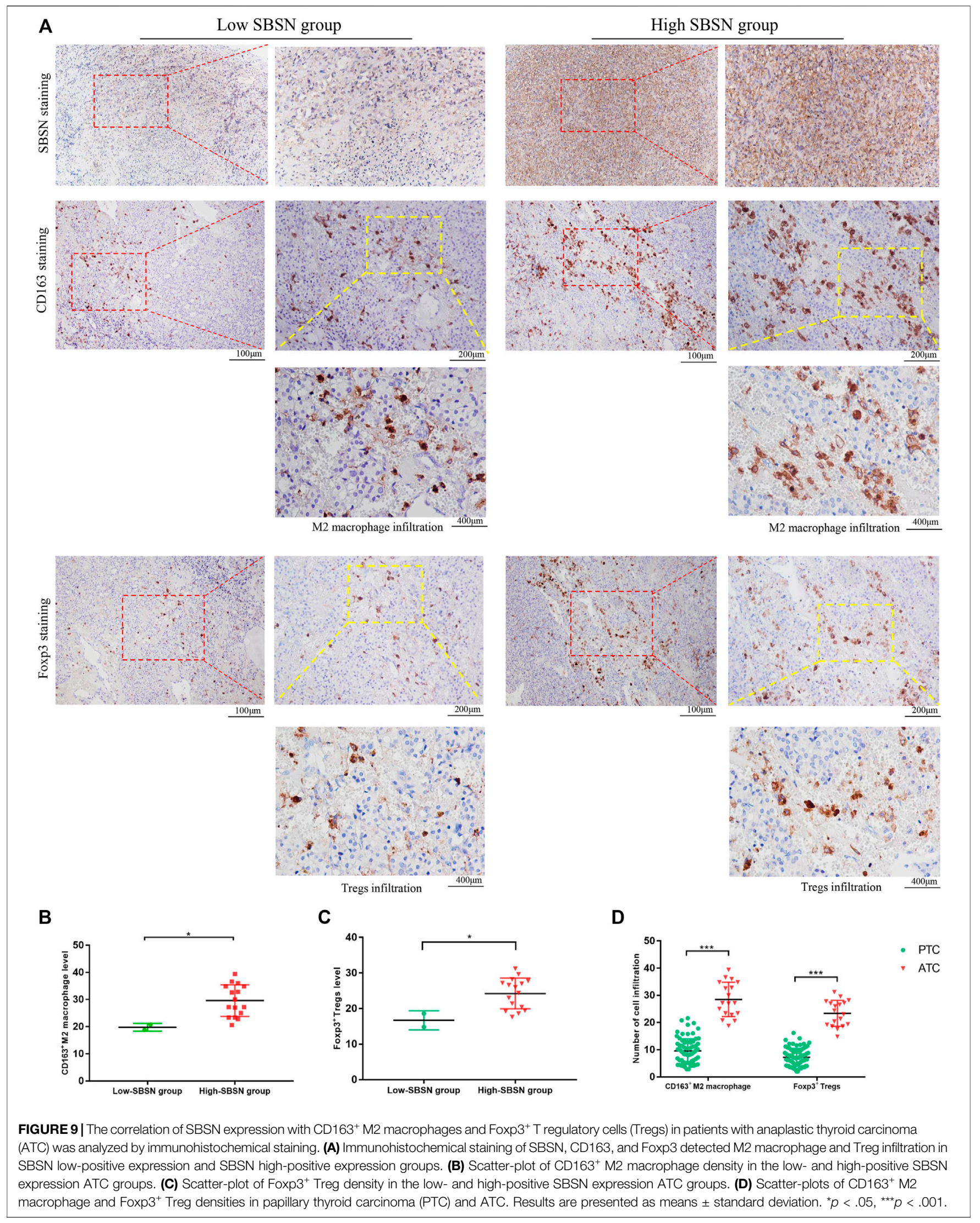




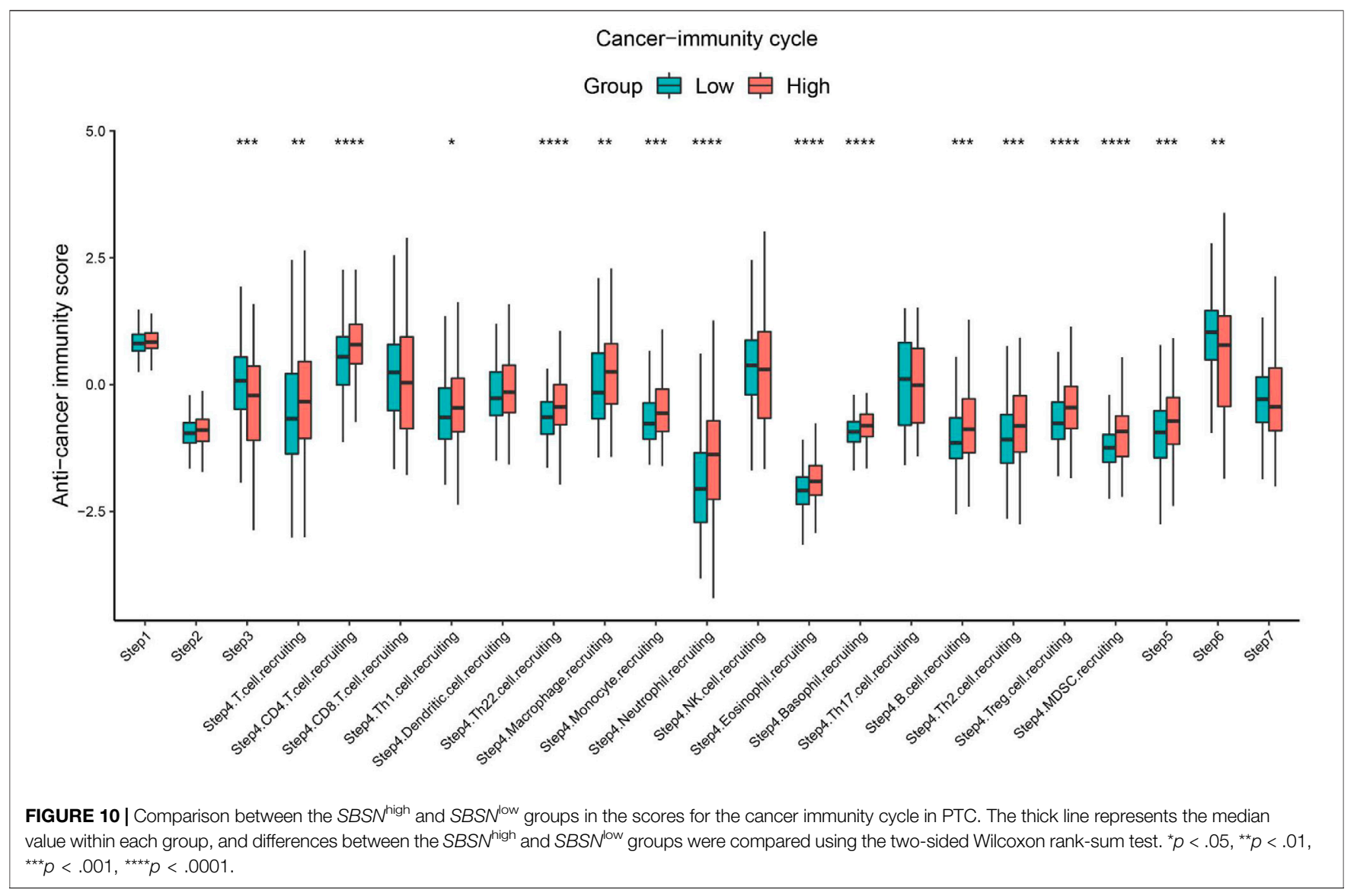

these results suggest that SBSN can regulate different types of tumor-associated cells in PTC as well as in the ATC TME.

\section{Correlation of SBSN Expression Levels in PTC and ATC Tumor Tissues With the Densities of CD163 ${ }^{+}$M2 Macrophages and Foxp $3^{+}$Tregs}

To confirm the results obtained by comprehensive bioinformatics analysis, we performed immunohistochemical staining of tumor tissues from 80 patients with PTC and 18 patients with ATC for the M2 macrophage marker CD163 and the Treg marker Foxp3. CD163+M2 macrophages and Foxp3+Tregs were mainly distributed in the interstitium of tumor tissues and lymphocyte aggregates in PTC (Figure 8A) and mainly scattered around cancer cells in ATC (Figure 9A). The results showed that, in PTC tissues, the infiltration levels of CD163+M2 macrophages (16.24 \pm $4.315)$ and Foxp3+Tregs $(11.88 \pm 2.581)$ were higher in the SBSN high-positive expression group than in the SBSN lowpositive expression group $(8.606 \pm 3.566$ and $6.566 \pm 2.69$, respectively; $p<.01$ ) (Figures $\mathbf{8 B}, \mathbf{C}$ ). In ATC tissues, the infiltration levels of CD163+M2 macrophages $(29.6 \pm 5.791)$ and Foxp $3+$ Tregs $(24.23 \pm 4.318)$ were higher in the SBSN highpositive expression group than in the SBSN low-positive expression group $(19.8 \pm 1.414$ and $16.7 \pm 2.687$, respectively; $p<.05$ ) (Figures 9B,C). In addition, the results of staining of CD163+M2 macrophages and Foxp3+Tregs in 80 cases of PTC and 18 of ATC showed that the total infiltration levels of CD163+M2 macrophages $(28.51 \pm 6.305)$ and Foxp3+Tregs $(23.39 \pm 4.775)$ were higher in ATC than in PTC $(9.56 \pm 4.417$ and $7.23 \pm 3.194$, respectively; $p<.01)$ (Figure 9D).

\section{Association of SBSN Expression With the Cancer Immunity Cycle}

The cancer immunity cycle involves a series of steps that enable the anti-cancer immune response to kill cancer cells effectively: tumor cell release of antigen (step I), tumor antigen presentation (step II), T cell activation (step III), $\mathrm{T}$ cell migration to tumor tissue (step IV), tumor tissue $\mathrm{T}$ cell infiltration (step V), T cell recognition of tumor cells (step VI), and clearance of tumor cells (step VII) (Chen and Mellman, 2013). The TIP pipeline was used to estimate the activity score of SBSN in the seven-step cancer immunity cycle in PTC samples. The results showed that the $S B S N^{\text {high }}$ group had higher scores $(p<.05)$ than those of the $S B S N^{\text {low }}$ group in the processes of $\mathrm{T}$ cell recruitment and infiltration of recruited $\mathrm{T}$ cells into tumor tissue. These included $\mathrm{CD}^{+}{ }^{+} \mathrm{T}$ cells, Th1 cells, Th22 cells, macrophages, monocytes, neutrophils, eosinophils, basophils, B cells, Th2 cells, Tregs, and bone 
marrow-derived suppressor cells (MDSCs). However, the $S B S N^{\text {high }}$ group had lower anti-cancer immune scores $(p<$ $.05)$ during T-cell activation and T-cell recognition of tumor cells (Figure 10). Overall, these results suggest a possible role of SBSN in the cancer immunity cycle of PTC.

\section{DISCUSSION}

This study aimed to predict the role of SBSN in follicular epithelial cell-derived thyroid cancer using an extensive bioinformatics data mining approach to explore the relationship between SBSN expression levels and the extent of infiltration by different immune cells. To our knowledge, the present study is the first to highlight the relationship between SBSN expression and immune infiltration in thyroid cancer, providing new insights into the role of SBSN in cancerassociated immune regulation and its application as a cancer biomarker.

SBSN was originally identified in epithelial tissues and is thought to be involved in the process of epidermal differentiation under physiological conditions (Park et al., 2002). In recent years, accumulating evidence has indicated that SBSN plays an important role in the development of a variety of tumors. Hypomethylation of the $S B S N$ promoter leads to elevated SBSN mRNA levels in NSCLC, and aberrant SBSN expression promotes the proliferation of lung squamous cell lines (Glazer et al., 2009). Similarly, in salivary ACC, the CpG island is hypomethylated in SBSN, and knockdown of $S B S N$ inhibits the proliferation and invasive ability of ACC cell lines (Shao et al., 2012). In ESCC, high SBSN expression level shortens patient survival, and overexpression of SBSN enhances the proliferation and invasive ability of esophageal cancer cells, while SBSN knockdown does the opposite (Zhu et al., 2016). In malignant brain tumors, SBSN upregulation is associated with poor prognosis for patients with glioblastoma multiforme (Formolo et al., 2011). Tumor development cannot be separated from angiogenesis, and SBSN is involved in the migration of tumor endothelial cells and angiogenesis through AKT activation (Alam et al., 2014; Takahashi et al., 2020). All of these previous findings suggest that $S B S N$ is involved as an oncogene in tumor progression.

In our study, the possible role of SBSN in follicular epithelial cell-derived thyroid cancer and the involved regulatory mechanisms were explored. SBSN was highly expressed in PTC at the protein and mRNA levels. SBSN expression was significantly associated with cervical-lymph-node metastasis in PTC $(p<.05)$. These results suggest that SBSN expression may be an indicator to assess the aggressiveness of PTC. Importantly, SBSN expression levels increased with decreasing extent of differentiation and increasing rates of malignancy in follicular epithelial cell-derived thyroid cancer; high-positive expression levels were most pronounced in ATC. This finding indicates that SBSN may be involved in regulating the malignancy of tumors and that its expression level may be related to the degree of tumor malignancy, accurately reflecting the progression of tumor malignancy.
We used two types of enrichment analysis to further elucidate the potential mechanisms of SBSN's involvement in follicularderived thyroid cancer. SBSN and its co-expressed genes were associated with a variety of biological processes, cellular components, and molecular functions, especially signaling, regulation, and responses to stimuli. SBSN was also found to be associated with several cancer signaling pathways, such as the $\mathrm{PI} 3 \mathrm{~K} / \mathrm{AKT}$ and MAPK pathways; this result is consistent with those of previous studies (Alam et al., 2014; Takahashi et al., 2020). Interestingly, SBSN expression was also significantly enriched in several cancer immunity-related pathways, such as the chemokine pathway, the cytokine and its receptor interaction pathway, and the $\mathrm{T}$-cell receptor signaling pathway, which are usually involved in tumorigenic processes (Liu T. et al., 2012; Li and Rudensky, 2016). These results suggest that SBSN may play complex roles in multiple biological processes.

A deeper analysis of the complexity within the TME may help identify patient populations with the potential to respond to current immune checkpoint therapies and may contribute to the identification of new adjuvant therapeutic targets (Lin et al., 2019). Previously, several studies have reported that elevated immune scores are associated with poor prognosis in patients with different cancers, such as renal cell carcinoma and osteosarcoma (Xu et al., 2019; Zhang et al., 2020), while stromal cells are also thought to play an important role in tumor growth, disease progression, and drug resistance (Denton et al., 2018). Therefore, we explored the relationship between TME scores and SBSN expression using the ESTIMATE algorithm. Our study is the first to demonstrate that, in PTC, SBSN expression level was positively correlated with immune scores and negatively correlated with tumor purity, while no significant association was observed between stromal scores and SBSN expression levels. In ATC, SBSN expression level was significantly positively correlated with immune and stromal scores and negatively correlated with tumor purity. These results suggest that SBSN may contribute to increasing numbers of immune and stromal cells in the TME, which diminishes tumor purity. Indeed, the elevated immune and stromal scores in the TME may indicate either protection or a poor prognosis for the host depending on the type of immune cells that infiltrate the tumor and the specific roles they play in tumor development (Cunha et al., 2014).

The types and relative proportions of TIICs in the TME may correlate with the clinical prognosis of patients (Orhan et al., 2020). Based on the functional enrichment analysis of SBSN and the correlation of its expression with immune and stromal cells in the TME, we further explored the relevance of SBSN expression to immune cell infiltration. Large amounts of RNA-seq data have enabled algorithms that employ the deconvolution principle or gene markers to map the TME of samples. Since there is no gold standard for inferring immune infiltration from RNA-seq data, we selected two algorithms, based on the deconvolution principle and the gene marker principle, to infer the composition of TIICs and confirmed their correlation by immunohistochemical staining. The proportion of M2 macrophages was higher in the $S B S N^{\text {high }}$ group in both the PTC and ATC samples. The proportion of M2 macrophages in the $S B S N^{\text {high }}$ group was much 
higher than that in the $S B S N^{\text {low }}$ group and higher than that of M1 macrophages in the $S B S N^{\text {high }}$ group in the ATC samples, suggesting that macrophages may be more polarized to the M2 phenotype in ATC. In most tumors, M2 macrophages are present as immunosuppressive cells, which can release growth factors to promote tumor development (Ho et al., 2016). More importantly, M2 macrophages tend to promote neoangiogenesis as well as stromal activation and remodeling (Afik et al., 2016), thus positively influencing cancer progression and negatively affecting patient prognosis (Tiainen et al., 2015). PTC tissues have been shown to have high levels of immunity, especially due to M2 macrophages, and compared with early PTC, advanced PTC exhibits a higher degree of immune infiltration and a higher proportion of M2 macrophages, which accelerate tumor cell migration (Zhang et al., 2021), producing a pro-cancer effect and exacerbating immune escape (Xie et al., 2020). Therefore, a high expression level of SBSN in patients with PTC and ATC may accelerate tumor progression by stimulating the polarization of M2 macrophages.

Furthermore, both CIBERSORT-ABS and xCell results showed that SBSN expression was positively correlated with the percentages of multiple DCs, including iDCs, and Tregs in PTC samples. Similarly, in ATC tissues, the percentage of iDCs was positively correlated with SBSN expression. The numbers of Tregs and DCs have been shown to increase in PTC tissues (Yu et al., 2013). Usually, tumor-infiltrating DCs exhibit an immature phenotype, resulting in altered antigen presentation (Tran Janco et al., 2015). iDCs do not effectively induce T- and NK-cellmediated immune responses and can even suppress immune responses by producing suppressive cytokines, such as IL-10 and TGF- $\beta$ (Scouten and Francis, 2006). In addition, Tregs, as well as TAMs, alter the normal replication of endothelial cells by creating a hypoxic environment in the tumor tissue and can achieve immunosuppressive and escape effects by inhibiting the antigen presentation by DCs and the activation of $\mathrm{CD}^{+}$ $\mathrm{T}$ cells in the tumor (Facciabene et al., 2017; Jang et al., 2017). The proportion of Tregs in PTC also correlates with lymph node metastasis and extrathyroidal expansion (French et al., 2010), which is consistent with our results. These findings suggest that patients with PTC and ATC with high SBSN expression levels may benefit from targeting SBSN to reduce the proportions of Tregs and iDCs.

In addition, SBSN expression levels in ATC samples were positively correlated with the proportions of mast cells and neutrophils, unlike those in PTC. The role of neutrophils in cancer is still controversial, but in recent years, thyroid cancer cells have been shown to be able to recruit neutrophils by releasing CXCL8/IL-8, improve their own survival by releasing granulocyte colony-stimulating factor, enhancing the proinflammatory response of neutrophils, and upregulate the expression of pro-oncogenic factors (Galdiero et al., 2018). Regarding mast cells in tumors, a previous study showed that the presence of mast cells in ATC correlates with tumor aggressiveness and that mast cells induce epithelialmesenchymal transition in thyroid cancer cell lines, mainly by activating CXCL8 in the AKT/SLUG pathway (Visciano et al., 2015). These results suggest that SBSN may be involved in the regulation of multiple infiltrating immune cells in the process of tumor development and contribute to tumor progression.

Interestingly, as suggested by the ESTIMATE algorithm, our exploration in PDTC yielded different results from those for ATC and PTC. CIBERSORT-ABS and xCell results showed a low level of immune cell infiltration in PDTC, and most immune cells and stromal cells did not correlate with SBSN expression. This is partly due to the small sample size of PDTC compared with those of PTC and ATC. However, we found similar results in the study by Giannini et al. (2019) who found that PDTC showed poor or absent immune cell infiltration compared with that in ATC and PTC, that the degree of immune cell infiltration in PDTC was even lower than that in normal thyroid tissue, and that, in most cases, PDTC appeared as non-T-cell-inflamed "cold" tumors.

Furthermore, our results showed that, in the xCell analysis of PTC, compared with the $S B S N^{\text {high }}$ group, the $S B S N^{\text {low }}$ group had a higher proportion of central memory $\mathrm{CD} 4^{+} \mathrm{T}$ cells as well as $\mathrm{CD}^{+}$naïve $\mathrm{T}$ cells. Central memory $\mathrm{CD} 4^{+} \mathrm{T}$ cells were reported to have stronger anti-tumor capacity compared with that of effector memory $\mathrm{CD}^{+} \mathrm{T}$ cells (Klebanoff et al., 2005). They have a strong self-renewal and replication ability and not only survive for a long time in vivo but also can be efficiently expanded in vitro to ensure the number of $\mathrm{T}$ cells returned for infusion, which can play a long-term anti-tumor role (Zhou et al., 2005; Berger et al., 2008). Naïve $\mathrm{CD}^{+} \mathrm{T}$ cells will differentiate into many effector cells when encountering antigens such as tumor cells, and these effector cells migrate to the corresponding sites to produce antitumor effects (Brummelman et al., 2018). The decrease in these cellular components coupled with an increase in SBSN expression indicates that the in vivo antitumor capacity is weakened and that tumor cells have a greater chance to develop immune escape ability.

Our results also demonstrated that some blood cells showed differential expression in the $S B S N^{\text {high }}$ and $S B S N^{\text {low }}$ groups. HSCs were able to promote tumor growth and progression in the solid TME (Hassan and Seno, 2020). However, the correlation between SBSN expression and HSCs in PTC and ATC produced opposite results, which may be attributed to the difference in the degree of malignancy of the two subtypes. Compared with PTC, ATC usually exhibits strong invasive and metastatic abilities, which are dependent on the supply of hematopoietic cells, reflecting the pro-carcinogenic role of SBSN in ATC. We also observed that the content of MEPs in ATC and PTC decreased with the expression of SBSN. Usually, in solid tumors or leukemia, MEPs can be transformed into erythroblast-like cells, erythrocytes, or megakaryocytes, thus promoting tumor progression (Wickrema and Crispino, 2007; Han et al., 2018). This implies that elevated SBSN expression may lead to increased conversion of MEPs into the aforementioned cells and may promote tumor progression.

In addition, studies on the dedifferentiation of thyroid cancer have gained interest in recent years. A previous study showed that immune scores are significantly negatively correlated with thyroid cancer differentiation scores ( $\mathrm{Na}$ and Choi, 2018) and that the least differentiated ATC usually has higher stromal and immune scores than those for highly differentiated PTC (Cunha et al., 2021), which suggests that the immune microenvironment 
may be involved in the process of thyroid cancer dedifferentiation. In our study, the xCell results showed that SBSN expression in ATC was positively correlated with fibroblast content in tumors, while no such relationship was found in PTC. Wen et al. (2021) found that cancer-associated fibroblast (CAF) content was significantly higher in ATC than in PTC or normal thyroid tissue. The content of CAFs was positively correlated with dedifferentiation, aggressiveness, and poor prognosis of thyroid cancer, which suggests that SBSN may promote dedifferentiation processes and contribute to poor outcomes of thyroid cancer by regulating the content of CAFs. In addition, M2 macrophages can activate the $\mathrm{Wnt} / \beta$-catenin pathway by secreting Wnt1 and Wnt3a, participating in the dedifferentiation, migration, and proliferation of invasive thyroid cancer cells (Lv et al., 2021). Our study indicated that SBSN expression level was positively correlated with the numbers of M2 macrophages in both PTC and ATC; however, compared with that in PTC, the proportion of M2 macrophages in the $S B S N^{\text {high }}$ ATC group was significantly higher than that in the $S B S N^{\text {low }}$ group. These findings suggest that SBSN may promote the dedifferentiation and aggressiveness of thyroid cancer cells by regulating the content of M2 macrophages, especially in ATC.

Regarding the cancer immunity cycle, patients with PTC with high SBSN expression levels scored higher in T-cell migration to tumor tissue (step IV) and tumor tissue T-cell infiltration (step V). However, according to the results of the cancer immunity cycle, most of the recruited and infiltrated immune cells, such as TAMs, neutrophils, and Tregs, have immunosuppressive effects on the tumor. There are also some helper T cells, such as Th2 cells, whose secreted cytokines can mediate the polarization of macrophages into the M2 phenotype (Shapouri-Moghaddam et al., 2018), and the increase in Th22 cell number has been reported to be associated with the progression of gastric cancer (Liu Z. et al., 2012). In addition, the recruitment of monocytes and MDSCs is positively correlated with SBSN expression in the cancer immunity cycle. Monocytes have been shown to be direct precursors of HSC-derived macrophages, and upon recruitment to tumor tissue, they can differentiate into TAMs and support tumorigenesis, local progression, and distant metastasis (Richards et al., 2013). MDSCs are rare in healthy subjects, but their numbers are elevated in patients with cancer, in whom they show a potent immunosuppressive potential and are associated with a poor prognosis (Marvel and Gabrilovich, 2015). Furthermore, patients with PTC with high SBSN expression levels showed lower scores in processes such as $\mathrm{T}$ cell activation and $\mathrm{T}$ cell recognition of tumor cells, which might be due to higher levels of iDCs and Tregs in their tissues, as DCs with an immature phenotype are usually unable to fully activate $\mathrm{T}$ cells (Yu et al., 2013) and Tregs also suppress the activation of $\mathrm{CD}^{+} \mathrm{T}$ cells (Jang et al., 2017). In addition, iDCs have abnormally altered antigen-presenting functions (Tran Janco et al., 2015), and Tregs in tumors also suppress antigenpresenting functions of DCs, leading to impaired T-cell recognition (Jang et al., 2017). These results imply that SBSN may suppress $\mathrm{T}$-cell activation and inhibit $\mathrm{T}$-cell recognition by affecting the levels of DCs and Tregs and may contribute to the immune escape of tumor cells.
There are several limitations to the current study. First, more thyroid cancer tissue samples are needed to validate the relationships between SBSN expression and immune and stromal cells in the TME and further explore the correlation between SBSN expression and immune cell infiltration. Second, there is no canonical method to analyze infiltrating immune cells in the TME, and we used two different methods that are based on different principles. Thus, additional studies are needed to elucidate the mechanism of SBSN's effects on immune cell infiltration in thyroid cancer. Third, RNA-seq-based algorithms may not be sufficiently accurate; overcoming this limitation requires in vivo models to explore the underlying biological mechanisms of SBSN's effects and its interaction with tumor immunity in thyroid cancer.

\section{CONCLUSION}

In conclusion, our study showed that SBSN is highly expressed in follicular epithelial cell-derived thyroid cancer. The expression level of SBSN increases with decreasing extent of cancer cell differentiation and is associated with lymph node metastasis in patients with PTC, which can be used as a potential biomarker for follicular epithelial cell-derived thyroid cancer. In addition, SBSN can influence the cancer immunity cycle and promote thyroid cancer dedifferentiation by regulating the level of immune cell infiltration in the TME. This suggests that SBSN may be a therapeutic target whose inhibition may promote anti-tumor immune response. Thus, a comprehensive understanding of the relationship between SBSN expression and immune infiltration may provide new insights into the immunotherapy of thyroid cancer.

\section{DATA AVAILABILITY STATEMENT}

The original contributions presented in the study are included in the article/Supplementary Material, further inquiries can be directed to the corresponding author.

\section{ETHICS STATEMENT}

The studies involving human participants were reviewed and approved by The Ethics Committee of the Shengjing Hospital, China Medical University. The patients/participants provided their written informed consent to participate in this study.

\section{AUTHOR CONTRIBUTIONS}

HT and ZL conceived and designed the experiments. HT collected the clinical data. HT, LW, and ZL analyzed and interpreted the data of the experiments. HT performed the experiments. The first draft was written by HT. LW participated in language editing. All authors read and approved the final manuscript. 


\section{FUNDING}

This work was supported by the 345 Talent Project of Shengjing Hospital of China Medical University.

\section{ACKNOWLEDGMENTS}

The authors would like to thank the investigators who contributed to this study. We thank all team members of TCGA, GSE29265, GSE33630, GSE76039, GSE65144, GSE53157, and GSE76039 projects for providing publicaccess data.

\section{REFERENCES}

Abdullah, M. I., Junit, S. M., Ng, K. L., Jayapalan, J. J., Karikalan, B., and Hashim, O. H. (2019). Papillary Thyroid Cancer: Genetic Alterations and Molecular Biomarker Investigations. Int. J. Med. Sci. 16 (3), 450-460. doi:10.7150/ijms. 29935

Afik, R., Zigmond, E., Vugman, M., Klepfish, M., Shimshoni, E., Pasmanik-Chor, M., et al. (2016). Tumor Macrophages Are Pivotal Constructors of Tumor Collagenous Matrix. J. Exp. Med. 213 (11), 2315-2331. doi:10.1084/jem. 20151193

Alam, M. T., Nagao-Kitamoto, H., Ohga, N., Akiyama, K., Maishi, N., Kawamoto, T., et al. (2014). Suprabasin as a Novel Tumor Endothelial Cell Marker. Cancer Sci. 105 (12), 1533-1540. doi:10.1111/cas.12549

Aoshima, M., Phadungsaksawasdi, P., Nakazawa, S., Iwasaki, M., Sakabe, J.-i., Umayahara, T., et al. (2019). Decreased Expression of Suprabasin Induces Aberrant Differentiation and Apoptosis of Epidermal Keratinocytes: Possible Role for Atopic Dermatitis. J. Dermatol. Sci. 95 (3), 107-112. doi:10.1016/j. jdermsci.2019.07.009

Aran, D., Hu, Z., and Butte, A. J. (2017). xCell: Digitally Portraying the Tissue Cellular Heterogeneity Landscape. Genome Biol. 18 (1), 220. doi:10.1186/ s13059-017-1349-1

Baxevanis, C. N., Sofopoulos, M., Fortis, S. P., and Perez, S. A. (2019). The Role of Immune Infiltrates as Prognostic Biomarkers in Patients with Breast Cancer. Cancer Immunol. Immunother. 68 (10), 1671-1680. doi:10.1007/s00262-01902327-7

Berger, C., Jensen, M. C., Lansdorp, P. M., Gough, M., Elliott, C., and Riddell, S. R. (2008). Adoptive Transfer of Effector CD8 $+\mathrm{T}$ Cells Derived from central Memory Cells Establishes Persistent T Cell Memory in Primates. J. Clin. Invest. 118 (1), 294-305. doi:10.1172/JCI32103

Brummelman, J., Pilipow, K., and Lugli, E. (2018). The Single-Cell Phenotypic Identity of Human CD8+ and CD4+ T Cells. Int. Rev. Cel Mol Biol. 341, 63-124. doi:10.1016/bs.ircmb.2018.05.007

Cancer Genome Atlas Research Network (2014). Integrated Genomic Characterization of Papillary Thyroid Carcinoma. Cell 159 (3), 676-690. doi:10.1016/j.cell.2014.09.050

Capdevila, J., Mayor, R., Mancuso, F. M., Iglesias, C., Caratù, G., Matos, I., et al. (2018). Early Evolutionary Divergence between Papillary and Anaplastic Thyroid Cancers. Ann. Oncol. 29 (6), 1454-1460. doi:10.1093/annonc/ mdy123

Chen, D. S., and Mellman, I. (2013). Oncology Meets Immunology: The CancerImmunity Cycle. Immunity 39 (1), 1-10. doi:10.1016/j.immuni.2013.07.012

Cunha, L. L., Domingues, G. A. B., Morari, E. C., Soares, F. A., Vassallo, J., and Ward, L. S. (2021). The Immune Landscape of the Microenvironment of Thyroid Cancer Is Closely Related to Differentiation Status. Cancer Cel Int. 21 (1), 387. doi:10.1186/s12935-021-02084-7

Cunha, L. L., Marcello, M. A., and Ward, L. S. (2014). The Role of the Inflammatory Microenvironment in Thyroid Carcinogenesis. Endocr. Relat. Cancer 21 (3), R85-R103. doi:10.1530/ERC-13-0431

\section{SUPPLEMENTARY MATERIAL}

The Supplementary Material for this article can be found online at: https://www.frontiersin.org/articles/10.3389/fgene.2022.810681/ full\#supplementary-material

Supplementary Figure S1 I (A) Survival curves of 502 patients with papillary thyroid carcinoma in the Cancer Genome Atlas (TCGA) database. (B) Comparison between $S B S N^{\text {high }}$ and $S B S N^{\text {low }}$ poorly differentiated thyroid carcinoma groups in the immune score, stromal score, estimate score, and tumor purity, calculated by ESTIMATE algorithm.

Supplementary Figure S2 | Non-cancerous cells that do not correlate with SBSN expression as derived by the xCell algorithm in papillary thyroid carcinoma.

Supplementary Figure $\mathbf{S 3}$ | Non-cancerous cells that do not correlate with SBSN expression as derived by the $\mathrm{xCell}$ algorithm in anaplastic thyroid carcinoma.

Denton, A. E., Roberts, E. W., and Fearon, D. T. (2018). Stromal Cells in the Tumor Microenvironment. Adv. Exp. Med. Biol. 1060, 99-114. doi:10.1007/978-3-31978127-3_6

Dralle, H., Machens, A., Basa, J., Fatourechi, V., Franceschi, S., Hay, I. D., et al. (2015). Follicular Cell-Derived Thyroid Cancer. Nat. Rev. Dis. Primers 1, 15077. doi:10.1038/nrdp.2015.77

Facciabene, A., De Sanctis, F., Pierini, S., Reis, E. S., Balint, K., Facciponte, J., et al. (2017). Local Endothelial Complement Activation Reverses Endothelial Quiescence, Enabling T-Cell Homing, and Tumor Control during T-Cell Immunotherapy. Oncoimmunology 6 (9), e1326442. doi:10.1080/2162402X. 2017.1326442

Fang, W., Ye, L., Shen, L., Cai, J., Huang, F., Wei, Q., et al. (2014). Tumorassociated Macrophages Promote the Metastatic Potential of Thyroid Papillary Cancer by Releasing CXCL8. Carcinogenesis 35 (8), 1780-1787. doi:10.1093/ carcin/bgu060

Formolo, C. A., Williams, R., Gordish-Dressman, H., MacDonald, T. J., Lee, N. H., and Hathout, Y. (2011). Secretome Signature of Invasive Glioblastoma Multiforme. J. Proteome Res. 10 (7), 3149-3159. doi:10.1021/pr200210w

French, J. D., Kotnis, G. R., Said, S., Raeburn, C. D., McIntyre, R. C., Klopper, J. P., et al. (2012). Programmed Death-1+ T Cells and Regulatory T Cells Are Enriched in Tumor-Involved Lymph Nodes and Associated with Aggressive Features in Papillary Thyroid Cancer. J. Clin. Endocrinol. Metab. 97 (6), E934-E943. doi:10.1210/jc.2011-3428

French, J. D., Weber, Z. J., Fretwell, D. L., Said, S., Klopper, J. P., and Haugen, B. R. (2010). Tumor-associated Lymphocytes and Increased Foxp3+ Regulatory T Cell Frequency Correlate with More Aggressive Papillary Thyroid Cancer. J. Clin. Endocrinol. Metab. 95 (5), 2325-2333. doi:10.1210/jc.2009-2564

Galdiero, M. R., Varricchi, G., Loffredo, S., Bellevicine, C., Lansione, T., Ferrara, A. L., et al. (2018). Potential Involvement of Neutrophils in Human Thyroid Cancer. PLoS ONE 13, e0199740. doi:10.1371/journal.pone.0199740

Giannini, R., Moretti, S., Ugolini, C., Macerola, E., Menicali, E., Nucci, N., et al. (2019). Immune Profiling of Thyroid Carcinomas Suggests the Existence of Two Major Phenotypes: An ATC-like and a PDTC-like. J. Clin. Endocrinol. Metab. 104 (8), 3557-3575. doi:10.1210/jc.2018-01167

Glazer, C. A., Smith, I. M., Ochs, M. F., Begum, S., Westra, W., Chang, S. S., et al. (2009). Integrative Discovery of Epigenetically Derepressed Cancer Testis Antigens in NSCLC. PLoS One 4 (12), e8189. doi:10.1371/journal.pone.0008189

Gospodarowicz, M., Mackillop, W., O'Sullivan, B., Sobin, L., Henson, D., Hutter, R. V., et al. (2001). Prognostic Factors in Clinical Decision Making: the Future. Cancer 91 (8 Suppl. 1), 1688-1695. doi:10.1002/1097-0142(20010415)91: $8+<1688$ :aid-cncr1184>3.0.co;2-7

Han, Y., Liu, Q., Hou, J., Gu, Y., Zhang, Y., Chen, Z., et al. (2018). Tumor-Induced Generation of Splenic Erythroblast-like Ter-Cells Promotes Tumor Progression. Cell 173 (3), 634-648. doi:10.1016/j.cell.2018.02.061

Hassan, G., and Seno, M. (2020). Blood and Cancer: Cancer Stem Cells as Origin of Hematopoietic Cells in Solid Tumor Microenvironments. Cells 9 (5), 1293. doi:10.3390/cells 9051293

Ho, V. W., Hofs, E., Elisia, I., Lam, V., Hsu, B. E., Lai, J., et al. (2016). All Trans Retinoic Acid, Transforming Growth Factor $\beta$ and Prostaglandin E2 in Mouse 
Plasma Synergize with Basophil-Secreted Interleukin-4 to M2 Polarize Murine Macrophages. PLoS One 11 (12), e0168072. doi:10.1371/journal.pone.0168072

Ichinose, K., Ohyama, K., Furukawa, K., Higuchi, O., Mukaino, A., Satoh, K., et al. (2018). Novel Anti-suprabasin Antibodies May Contribute to the Pathogenesis of Neuropsychiatric Systemic Lupus Erythematosus. Clin. Immunol. 193, 123-130. doi:10.1016/j.clim.2017.11.006

Jang, J.-E., Hajdu, C. H., Liot, C., Miller, G., Dustin, M. L., and Bar-Sagi, D. (2017). Crosstalk between Regulatory T Cells and Tumor-Associated Dendritic Cells Negates Anti-tumor Immunity in Pancreatic Cancer. Cel Rep. 20 (3), 558-571. doi:10.1016/j.celrep.2017.06.062

Jung, K. Y., Cho, S. W., Kim, Y. A., Kim, D., Oh, B.-C., Park, D. J., et al. (2015). Cancers with Higher Density of Tumor-Associated Macrophages Were Associated with Poor Survival Rates. J. Pathol. Transl Med. 49 (4), 318-324. doi:10.4132/jptm.2015.06.01

Klebanoff, C. A., Gattinoni, L., Torabi-Parizi, P., Kerstann, K., Cardones, A. R., Finkelstein, S. E., et al. (2005). Central Memory Self/tumor-Reactive CD8+ T Cells Confer superior Antitumor Immunity Compared with Effector Memory T Cells. Proc. Natl. Acad. Sci. 102 (27), 9571-9576. doi:10.1073/pnas. 0503726102

La Vecchia, C., Malvezzi, M., Bosetti, C., Garavello, W., Bertuccio, P., Levi, F., et al. (2015). Thyroid Cancer Mortality and Incidence: a Global Overview. Int. J. Cancer 136 (9), 2187-2195. doi:10.1002/ijc.29251

Landa, I., Ibrahimpasic, T., Boucai, L., Sinha, R., Knauf, J. A., Shah, R. H., et al. (2016). Genomic and Transcriptomic Hallmarks of Poorly Differentiated and Anaplastic Thyroid Cancers. J. Clin. Invest. 126 (3), 1052-1066. doi:10.1172/ JCI85271

Li, M. O., and Rudensky, A. Y. (2016). T Cell Receptor Signalling in the Control of Regulatory T Cell Differentiation and Function. Nat. Rev. Immunol. 16 (4), 220-233. doi:10.1038/nri.2016.26

Lin, P., Guo, Y.-n., Shi, L., Li, X.-j., Yang, H., He, Y., et al. (2019). Development of a Prognostic index Based on an Immunogenomic Landscape Analysis of Papillary Thyroid Cancer. Aging 11 (2), 480-500. doi:10.18632/aging.101754

Liu, T., Peng, L., Yu, P., Zhao, Y., Shi, Y., Mao, X., et al. (2012a). Increased Circulating Th22 and Th17 Cells Are Associated with Tumor Progression and Patient Survival in Human Gastric Cancer. J. Clin. Immunol. 32 (6), 1332-1339. doi:10.1007/s10875-012-9718-8

Liu, Z., Sun, D.-X., Teng, X.-Y., Xu, W.-X., Meng, X.-P., and Wang, B.-S. (2012b). Expression of Stromal Cell-Derived Factor 1 and CXCR7 in Papillary Thyroid Carcinoma. Endocr. Pathol. 23 (4), 247-253. doi:10.1007/s12022-012-9223-x

Lote, H., Cafferkey, C., and Chau, I. (2015). PD-1 and PD-L1 Blockade in Gastrointestinal Malignancies. Cancer Treat. Rev. 41 (10), 893-903. doi:10. 1016/j.ctrv.2015.09.004

Lv, J., Feng, Z. P., Chen, F. K., Liu, C., Jia, L., Liu, P. J., et al. (2021). M2-like Tumorassociated Macrophages-secreted Wnt1 and Wnt3a Promotes Dedifferentiation and Metastasis via Activating $\beta$-catenin Pathway in Thyroid Cancer. Mol. Carcinogenesis 60 (1), 25-37. doi:10.1002/mc.23268

Marvel, D., and Gabrilovich, D. I. (2015). Myeloid-derived Suppressor Cells in the Tumor Microenvironment: Expect the Unexpected. J. Clin. Invest. 125 (9), 3356-3364. doi:10.1172/JCI80005

Mazzaferri, E. L., and Jhiang, S. M. (1994). Long-term Impact of Initial Surgical and Medical Therapy on Papillary and Follicular Thyroid Cancer. Am. J. Med. 97 (5), 418-428. doi:10.1016/0002-9343(94)90321-2

Melillo, R. M., Guarino, V., Avilla, E., Galdiero, M. R., Liotti, F., Prevete, N., et al. (2010). Mast Cells Have a Protumorigenic Role in Human Thyroid Cancer. Oncogene 29 (47), 6203-6215. doi:10.1038/onc.2010.348

Molinaro, E., Romei, C., Biagini, A., Sabini, E., Agate, L., Mazzeo, S., et al. (2017). Anaplastic Thyroid Carcinoma: from Clinicopathology to Genetics and Advanced Therapies. Nat. Rev. Endocrinol. 13 (11), 644-660. doi:10.1038/ nrendo.2017.76

Motzer, R. J., Escudier, B., McDermott, D. F., George, S., Hammers, H. J., Srinivas, S., et al. (2015). Nivolumab versus Everolimus in Advanced Renal-Cell Carcinoma. N. Engl. J. Med. 373 (19), 1803-1813. doi:10.1056/NEJMoa 1510665

$\mathrm{Na}, \mathrm{K}$. J., and Choi, H. (2018). Immune Landscape of Papillary Thyroid Cancer and Immunotherapeutic Implications. Endocr. Relat. Cancer 25 (5), 523-531. doi:10.1530/ERC-17-0532

Newman, A. M., Liu, C. L., Green, M. R., Gentles, A. J., Feng, W., Xu, Y., et al. (2015). Robust Enumeration of Cell Subsets from Tissue Expression Profiles. Nat. Methods 12 (5), 453-457. doi:10.1038/nmeth.3337
Orhan, A., Vogelsang, R. P., Andersen, M. B., Madsen, M. T., Hölmich, E. R., Raskov, H., et al. (2020). The Prognostic Value of Tumour-Infiltrating Lymphocytes in Pancreatic Cancer: a Systematic Review and Meta-Analysis. Eur. J. Cancer 132, 71-84. doi:10.1016/j.ejca.2020.03.013

Papp, S., and Asa, S. L. (2015). When Thyroid Carcinoma Goes Bad: a Morphological and Molecular Analysis. Head Neck Pathol. 9 (1), 16-23. doi:10.1007/s12105-015-0619-z

Park, G. T., Lim, S. E., Jang, S.-I., and Morasso, M. I. (2002). Suprabasin, a Novel Epidermal Differentiation Marker and Potential Cornified Envelope Precursor. J. Biol. Chem. 277 (47), 45195-45202. doi:10.1074/jbc.m205380200

Reck, M., Rodríguez-Abreu, D., Robinson, A. G., Hui, R., Csőszi, T., Fülöp, A., et al. (2016). Pembrolizumab versus Chemotherapy for PD-L1-Positive Non-smallcell Lung Cancer. N. Engl. J. Med. 375 (19), 1823-1833. doi:10.1056/ NEJMoa1606774

Richards, D. M., Hettinger, J., and Feuerer, M. (2013). Monocytes and Macrophages in Cancer: Development and Functions. Cancer Microenvironment 6 (2), 179-191. doi:10.1007/s12307-012-0123-x

Scouten, W. T., and Francis, G. L. (2006). Thyroid Cancer and the Immune System: a Model for Effective Immune Surveillance. Expert Rev. Endocrinol. Metab. 1 (3), 353-366. doi:10.1586/17446651.1.3.353

Shao, C., Tan, M., Bishop, J. A., Liu, J., Bai, W., Gaykalova, D. A., et al. (2012). Suprabasin Is Hypomethylated and Associated with Metastasis in Salivary Adenoid Cystic Carcinoma. PLoS One 7 (11), e48582. doi:10.1371/journal.pone. 0048582

Shapouri-Moghaddam, A., Mohammadian, S., Vazini, H., Taghadosi, M., Esmaeili, S. A., Mardani, F., et al. (2018). Macrophage Plasticity, Polarization, and Function in Health and Disease. J. Cel Physiol. 233 (9), 6425-6440. doi:10. $1002 /$ jcp. 26429

Takahashi, K., Asano, N., Imatani, A., Kondo, Y., Saito, M., Takeuchi, A., et al. (2020). Sox2 Induces Tumorigenesis and Angiogenesis of Early-Stage Esophageal Squamous Cell Carcinoma through Secretion of Suprabasin. Carcinogenesis 41 (11), 1543-1552. doi:10.1093/carcin/bgaa014

Tiainen, S., Tumelius, R., Rilla, K., Hämäläinen, K., Tammi, M., Tammi, R., et al. (2015). High Numbers of Macrophages, Especially M2-like (CD163-Positive), Correlate with Hyaluronan Accumulation and Poor Outcome in Breast Cancer. Histopathology 66 (6), 873-883. doi:10.1111/his.12607

Tran Janco, J. M., Lamichhane, P., Karyampudi, L., and Knutson, K. L. (2015). Tumor-infiltrating Dendritic Cells in Cancer Pathogenesis. J.I. 194 (7), 2985-2991. doi:10.4049/jimmunol.1403134

Visciano, C., Liotti, F., Prevete, N., Cali', G., Franco, R., Collina, F., et al. (2015). Mast Cells Induce Epithelial-To-Mesenchymal Transition and Stem Cell Features in Human Thyroid Cancer Cells through an IL-8-Akt-Slug Pathway. Oncogene 34 (40), 5175-5186. doi:10.1038/onc.2014.441

Waniczek, D., Lorenc, Z., Śnietura, M., Wesecki, M., Kopec, A., and MucWierzgoń, M. (2017). Tumor-Associated Macrophages and Regulatory $\mathrm{T}$ Cells Infiltration and the Clinical Outcome in Colorectal Cancer. Arch. Immunol. Ther. Exp. 65 (5), 445-454. doi:10.1007/s00005-017-0463-9

Wen, S., Qu, N., Ma, B., Wang, X., Luo, Y., Xu, W., et al. (2021). Cancer-Associated Fibroblasts Positively Correlate with Dedifferentiation and Aggressiveness of Thyroid Cancer. Ott 14, 1205-1217. doi:10.2147/OTT.S294725

Wickrema, A., and Crispino, J. D. (2007). Erythroid and Megakaryocytic Transformation. Oncogene 26 (47), 6803-6815. doi:10.1038/sj.onc.1210763

Xiangqian, Z., Chen, P., Ming, G., Jingtai, Z., Xiukun, H., Jingzhu, Z., et al. (2019). Risk Factors for Cervical Lymph Node Metastasis in Papillary Thyroid Microcarcinoma: a Study of 1,587 Patients. Cancer Biol. Med. 16 (1), 121-130. doi:10.20892/j.issn.2095-3941.2018.0125

Xie, Z., Li, X., He, Y., Wu, S., Wang, S., Sun, J., et al. (2020). Immune Cell Confrontation in the Papillary Thyroid Carcinoma Microenvironment. Front. Endocrinol. 11, 570604. doi:10.3389/fendo.2020.570604

Xu, B., and Ghossein, R. (2020). Poorly Differentiated Thyroid Carcinoma. Semin. Diagn. Pathol. 37 (5), 243-247. doi:10.1053/j.semdp.2020.03.003

Xu, L., Deng, C., Pang, B., Zhang, X., Liu, W., Liao, G., et al. (2018). TIP: A Web Server for Resolving Tumor Immunophenotype Profiling. Cancer Res. 78 (23), 6575-6580. doi:10.1158/0008-5472.CAN-18-0689

Xu, W.-H., Xu, Y., Wang, J., Wan, F.-N., Wang, H.-K., Cao, D.-L., et al. (2019). Prognostic Value and Immune Infiltration of Novel Signatures in clear Cell Renal Cell Carcinoma Microenvironment. Aging 11 (17), 6999-7020. doi:10. 18632/aging.102233 
Yoshihara, K., Shahmoradgoli, M., Martínez, E., Vegesna, R., Kim, H., TorresGarcia, W., et al. (2013). Inferring Tumour Purity and Stromal and Immune Cell Admixture from Expression Data. Nat. Commun. 4, 2612. doi:10.1038/ ncomms 3612

Yu, H., Huang, X., Liu, X., Jin, H., Zhang, G. e., Zhang, Q., et al. (2013). Regulatory T Cells and Plasmacytoid Dendritic Cells Contribute to the Immune Escape of Papillary Thyroid Cancer Coexisting with Multinodular Non-toxic Goiter. Endocrine 44 (1), 172-181. doi:10.1007/s12020-012-9853-2

Zhang, C., Gu, X., Pan, M., Yuan, Q., and Cheng, H. (2021). Senescent Thyroid Tumor Cells Promote Their Migration by Inducing the Polarization of M2-like Macrophages. Clin. Transl Oncol. 23 (6), 1253-1261. doi:10.1007/s12094-02002516-2

Zhang, C., Zheng, J.-H., Lin, Z.-H., Lv, H.-Y., Ye, Z.-M., Chen, Y.-P., et al. (2020). Profiles of Immune Cell Infiltration and Immune-Related Genes in the Tumor Microenvironment of Osteosarcoma. Aging 12 (4), 3486-3501. doi:10.18632/ aging.102824

Zhang, H., Liu, H., Shen, Z., Lin, C., Wang, X., Qin, J., et al. (2018). Tumorinfiltrating Neutrophils Is Prognostic and Predictive for Postoperative Adjuvant Chemotherapy Benefit in Patients with Gastric Cancer. Ann. Surg. 267 (2), 311-318. doi:10.1097/SLA.0000000000002058

Zhou, J., Dudley, M. E., Rosenberg, S. A., and Robbins, P. F. (2005). Persistence of Multiple Tumor-specific T-Cell Clones Is Associated with Complete Tumor
Regression in a Melanoma Patient Receiving Adoptive Cell Transfer Therapy. J. Immunother. 28 (1), 53-62. doi:10.1097/00002371-200501000-00007

Zhu, J., Wu, G., Li, Q., Gong, H., Song, J., Cao, L., et al. (2016). Overexpression of Suprabasin Is Associated with Proliferation and Tumorigenicity of Esophageal Squamous Cell Carcinoma. Sci. Rep. 6, 21549. doi:10.1038/srep21549

Conflict of Interest: The authors declare that the research was conducted in the absence of any commercial or financial relationships that could be construed as a potential conflict of interest.

Publisher's Note: All claims expressed in this article are solely those of the authors and do not necessarily represent those of their affiliated organizations, or those of the publisher, the editors and the reviewers. Any product that may be evaluated in this article, or claim that may be made by its manufacturer, is not guaranteed or endorsed by the publisher.

Copyright (c) 2022 Tan, Wang and Liu. This is an open-access article distributed under the terms of the Creative Commons Attribution License (CC BY). The use, distribution or reproduction in other forums is permitted, provided the original author(s) and the copyright owner(s) are credited and that the original publication in this journal is cited, in accordance with accepted academic practice. No use, distribution or reproduction is permitted which does not comply with these terms. 